\title{
ALGORITHMS AND THE STREAMING WARS: THE CHANGING MEANINGS OF FILM AND TELEVISION CULTURE
}

\author{
By \\ Niko Pajkovic \\ Honours Bachelour of Arts in Communication Studies \\ Wilfrid Laurier University, 2017 \\ A MRP presented by Ryerson University \\ in partial fulfillment of \\ the requirements for the degree of \\ Master of Arts \\ in the program of Professional Communication
}

Toronto, Ontario, Canada, 2020

(C) Niko Pajkovic 


\section{Author's Declaration for Electronic Submission of a Major Research Paper}

I hereby declare that I am the sole author of this Major Research Paper and the accompanying Research Poster. This is a true copy of the MRP and the research poster, including any required final revisions, as accepted by my examiners.

I authorize Ryerson University to lend this major research paper and/or poster to other institutions or individuals for the purpose of scholarly research.

I further authorize Ryerson University to reproduce this MRP and/or poster by photocopying or by other means, in total or in part, at the request of other institutions or individuals for the purpose of scholarly research.

I understand that my MRP and/or my MRP research poster may be made electronically available to the public. 


\begin{abstract}
The film and television industry has been transformed by a new wave of over-the-top (OTT) video streaming services. Disney+, Apple TV Plus, NBC Universal's Peacock, WarnerMedia's HBO Max, and Quibi have all been released between November 12th, 2019 and May 27th, 2020, ushering in what the media has called the "Streaming Wars". Like Netflix, Amazon, and Hulu, these platforms are dependent on the use of algorithms and Big Data, meaning the presence of these technologies within the industry will become increasingly pervasive, important, and unavoidable for producers and consumers alike. The purpose of this MRP is twofold: 1) to explore the current role algorithms play in the production, distribution and consumption of film and television, and to assess how these technologies are impacting broader notions of creativity and taste within the industry; and 2) to challenge the dominant critical theoretical perspectives that have emerged in regards to algorithmic cultures, namely, those contending that algorithms are replacing the fundamentally human process of cultural meaning- and decision-making. To achieve this, I explore the role algorithms play in the production and creative development of film and television, focusing my analysis on the emergence of data-driven creativity. I examine several third-party AI and analytics firms whose services automate the creative practices of ideation, script development, and casting. In addition, I examine how algorithms are changing the distribution and consumption of film and television via recommendation systems, and contribute to the existing dialogue regarding their implications on taste and taste-making. For that purpose, I apply Bucher's (2018) method of reverse engineering to the Netflix Recommender System (NRS), revealing its circular and economic logics.
\end{abstract}




\section{Table of Contents}

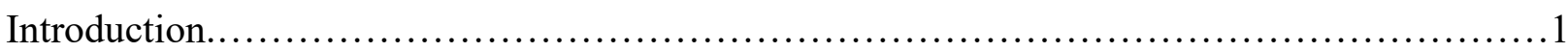

Literature Review..............................................................6

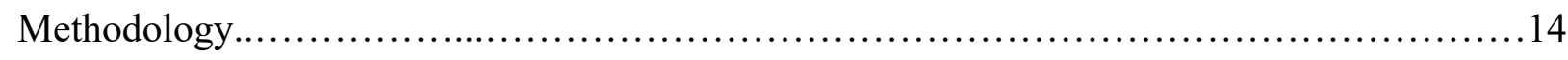

The Streaming Wars............................................................ 18

Data-Driven Creativity and Networked Cultural Production..............................26

Exposing the Operational Logics of the Netflix Recommender System .................. 43

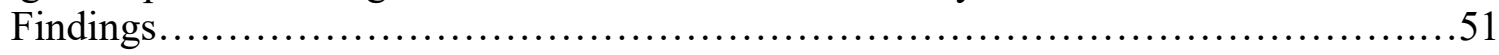

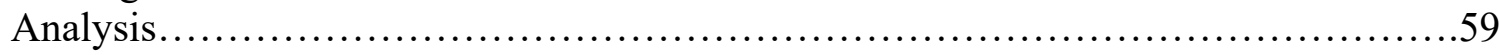

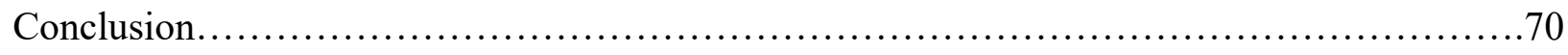

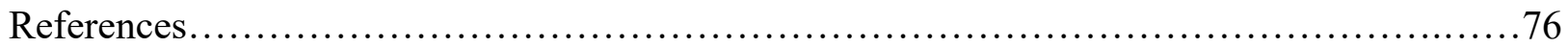




\title{
Algorithms and the "Streaming Wars": The Changing Meanings of Film and Television Culture
}

\begin{abstract}
You have 'to follow the actors themselves', that is try to catch up with their often wild innovations in order to learn from them what the collective existence has become in their hands, which methods they have elaborated to make it fit together, which accounts could best define the new associations that they have been forced to establish. (Latour, 2015, p. 12)
\end{abstract}

The ubiquitous use of algorithmic technology today raises a series of concerns for society and researchers alike. The functioning of entire industries is now built on top of these intricate and often invisible technologies, from finance to journalism, entertainment to healthcare, and manufacturing to media and technology. Furthermore, the public - knowingly or not - is becoming increasingly dependent on algorithms in their everyday practices. Algorithms sort and order the information we consume, secure and encrypt our personal data, recommend us consumer goods and cultural products, predict our preferences and habits, and auto-correct our errors; they optimize and inform our inputs, and have an often imperceptible impact on thousands of other routine elements of everyday life. Roberge and Seyfert (2016) go so far as to argue that "no area of human experience is untouched by them" (p. 1). As a result, a new field of study has emerged within the social sciences and humanities routinely referred to as Critical Algorithm Studies (Social Media Collective, 2016). A majority of the research within this field has focused on the capacity for algorithms to (re)produce systems of power, governance, and surveillance (see, e.g., Beer, 2009; Mager, 2012; Pasque, 2015). However, a smaller, but steadily growing subset of this research has turned its attention specifically to the relationship between algorithms and culture. Researchers of algorithmic cultures frequently question the power algorithms have to sort, rank, and distribute cultural items, and therefore shape our cultural tastes, preferences, and practices (see, e.g., Beer, 2013; Morris, 2015; Striphas, 2015). This MRP 
will contribute to this growing area of research by unpacking the complex and multilayered role algorithms now play in the production, distribution, and consumption of film and television.

Focusing on film and television as a site of cultural inquiry in order to better understand the cultural functions of algorithms has proved to be a pertinent research endeavour. The industry has recently been transformed by a new wave of over-the-top (OTT) video streaming services, all of which heavily rely on the use of algorithms. Disney+, Apple TV Plus, NBC Universal's Peacock, WarnerMedia's HBO Max, and Quibi have all been released between November 12th, 2019 and May 27th, 2020, ushering in what the media has called the "Streaming Wars". In addition, the COVID-19 pandemic and corresponding global lockdowns have only accelerated this evolutionary shift taking place in the film and television industry; the pandemic has prompted a significant increase in the global consumption of online film and television content (Economist, 2020), it has led to the proliferation of "virtual cinemas" and online film festivals (Erbland, 2020), and it has intensified the competitive dynamics of the Streaming Wars, allowing services like Netflix and Disney+ to amass record-breaking subscriber numbers (Spangler, 2020). As these services - all of which deliver their own impressive catalogues of films and shows - compete for subscriptions, what ends up attracting potential users to one service over the other may depend not on the actual content they offer, so much as the ways that that content is produced, organized, and recommended by algorithmic technology. If this is the case, the industry will witness an acceleration in the strategic development and implementation of algorithms, which will in turn make their presence increasingly important, pervasive, and unavoidable for consumers hungry for relevant and stimulating content. This shift also has the potential to exacerbate the concerns already raised by researchers of algorithmic cultures, namely that algorithms are replacing the fundamentally human - or at least, the less digitally mediated - process of cultural meaning- and decision-making. 
From a research perspective, I see the transformative shift taking place in the film and television industry today as presenting a prime opportunity to evaluate the evolving operations of algorithms and their entanglements with culture. Furthermore, I view this shift as offering researchers an appropriate moment to critically assess the existing scholarship on algorithmic cultures and the dominant perspectives that have emerged from it. Amongst these perspectives there is a recurring tendency to think of algorithms as "black boxed" technical devices that possess the power to shape cultural tastes and practices, while remaining exogenous from culture itself. I do not intend to criticize these perspectives, as many have offered important contributions to the study of algorithmic cultures and provided useful ideas and frameworks for my own research; however, I want to suggest that the tendency of these perspectives to understand algorithms as though they were one-way actors in our culture has amplified rather than eliminated the need for more holistic and multilayered analyses, particularly those that take into consideration the network of actors involved in an algorithm's operation, the numerous ways that algorithms are interacted with, and the many layers of existence and meaning they take on. In order to examine the multilayered role algorithms play in the production, distribution, and consumption of film and television, I will adopt a relational materialist perspective of algorithmic technology, as developed by scholars such as Roberge and Seyfert (2016), Kitchin (2017), Seaver (2017), and Bucher (2018).

From a relational materialist perspective, algorithms are understood to be socio-technical processes that come into existence and operate in the world via a series of complex relations between human and non-human actors. As Kitchin (2017) puts it, we need to conceive of algorithms "as being contingent, ontogenetic, performative in nature and embedded in wider socio-technical assemblages" (p. 16). This perspective argues that algorithms are not static technical objects transforming culture from the outside, but rather dynamic and evolving 
processes that are enacted by a combination of social, cultural, and technical practices that themselves respond to culture as they shape it. They are defined by their "constitutive entanglement" (Introna \& Hayes 2011, p. 108) with the multiplicity of actors involved in their becoming, all of whom serve varying purposes and are motivated by different interests and desires. These actors can include the algorithm's code and the data structures they work on, other software and systems of algorithms that they engage with, the programmers and engineers who construct them, the companies and institutions that deploy them, the regulators who oversee them, the users who interact with them, the broader public who articulate and make sense of their presence, and the complex contexts that inform the contingent nature of their becoming.

The goal of this MRP is not simply to report on the ways algorithms are being used in the film and television industry, or to accuse algorithms of denuding culture; rather I intend to draw attention to the ways algorithms are deeply entangled with culture, and to ultimately question how these entanglements work to alter the very meaning of film and television, creativity, taste, and culture more broadly. To do so, I have adopted Bucher's $(2016,2018)$ innovative and flexible methodology of technography and my paper is divided in the following way: 1) I provide an overview of the current OTT ecosystem; 2) I explore the role algorithms play in the production and creative development of film and television, focusing my analysis on the emergence of data-driven creativity. I examine several third-party AI and analytics firms whose services automate the creative practices of ideation, script development, and casting; and 3) I examine how algorithms are changing the distribution and consumption of film and television via recommendation systems, and contribute to the existing dialogue regarding their implications on taste and taste-making. In order to do so, I apply Bucher's (2018) method of reverse engineering to the Netflix Recommender System (NRS), revealing its circular and economic logics. 
Although each of these elements could arguably make up their own papers, by addressing them all I hope to offer a more comprehensive analysis of the multilayered role algorithms are playing in the production, distribution, and consumption of film and television, one that embraces, instead of struggles against, the relational, contingent, and ontogenetic nature of algorithms (Kitchin, 2017). By taking this approach I am also able to avoid the risk of narrowing down algorithms, or equally complex concepts such as taste, creativity, and culture, to a single social phenomenon that can be analyzed and measured via one specific method. This paper is far more concerned with expanding the conversation and connecting dots than it is with reaching fixed conclusions or discovering precise answers.

Before proceeding I want to make it clear that throughout this paper, I use the term algorithm as synecdoche for the "ill-defined network" that combines "algorithm, model, target goal, data, training data, application, hardware" (Gillispie, 2016b). While I make distinctions between Big Data or data analytics, from algorithms or algorithmic technologies (e.g. machine learning and $\mathrm{AI}$ ), these are all objects and processes that are inextricably tied to one another. In order to collect and make sense of large data sets one needs algorithms, and for algorithms to exist they need input data in order to operate.

\section{Research Questions}

This paper will engage with the following research questions:

1) What can the existence of third-party firms like Vault AI, ScriptBook, and Cinelytic tell us about the role Big Data and algorithmic technology now play in the development and production of film and television and how might this shift to data-driven and algorithmically-led production impact creativity?

2) What are the operational logics of the Netflix Recommender System and what new forms of meaning could video recommender systems, like the NRS, produce in regards to taste? 


\section{Literature Review}

\section{Relational materialism: Algorithms as sociotechnical processes}

Bucher $(2016,2018)$ argues that researchers in the social science and humanities tend to place too much emphasis on issues of expertise, access, and transparency when discussing algorithms. Due to their technical complexity, as well as the fact that their code and other details are routinely proprietary and kept secret by the corporations and institutions that use them, algorithms have been repeatedly referred to as "black box" devices. This metaphor positions algorithms as opaque technical objects "whose inner functioning cannot be known" (Bucher, 2018, p. 43). Therefore, a pressing area of concern for researchers has been the way algorithmic secrecy can be used for financial, political, and legal gain (see, e.g, Frank Pasquale, 2015). For Bucher, the black box metaphor and the critical perspectives inclined to adopt it, do not present an adequate way of thinking about the ontology and epistemology of algorithms. She suggests that viewing algorithms as powerful, yet unknowable entities whose core essence is locked in their code, ignores the inherent multiplicity of their existence and deters quality research from being conducted. For Bucher, algorithms are "entangled, multiple, and eventful, and therefore, things that cannot be understood as being powerful in one way only" (Bucher, 2018, p. 20). She suggests that a first step towards better understanding algorithms is, paradoxically, to un-know them; to challenge the existing ways that they are known, to engage with the qualities of algorithms that cannot be expressed in fully rational terms, and to open up the "black box of one's own assumptions" about them (Bucher, 2018, p. 46).

Seaver (2017) proposes an ethnographic approach to studying algorithms. He stresses the need to try to understand how algorithms work in practice, through fieldwork. Crucial to this approach he argues, is entering the field without preconceived ideas of what algorithms are, especially in technical terms. Like Bucher, Seaver regards algorithms as heterogenous and 
distributed socio-technical systems, rather than as "rigidly constrained and procedural formulas" (Seaver, 2017, p. 1). He offers two anthropological perspectives of algorithms: algorithms in culture and algorithms as culture. An anthropological perspective of algorithms in culture rests on the notion that "algorithms are discrete objects that may be located within cultural contexts or brought into conversation with cultural concerns" (Seaver, 2017, p. 4). Here, culture and algorithms are seen as being fundamentally separate and therefore able to influence one another. Seaver rejects this perspective and endorses the second view of algorithms as culture, which sees algorithms "as part of culture, constituted not only by rational procedures, but by institutions, people, intersecting contexts, and the rough-and-ready sensemaking that obtains in ordinary cultural life views" (p. 10). In his analysis of Twitter's trending algorithm Gillespie (2016a) also argues that when researching algorithms "we should ask not just how algorithms shape culture, but how they become culture" (p. 54).

Similarly, Roberge and Seyfert (2017) argue that algorithms are both anthropologically and temporally entrenched, unfolding in "a state of incessant negotiation and in-betweenness" (p. 2). What these authors are drawing attention to is the symbiotic relationship between algorithms and humans, whose mutual engagement with one another leaves both in a constant state of change and evolution via input/output feedback loops. Due to this, they argue that the agency of algorithms "can best be described as 'fractal"', and their ability to control cultural processes is in actuality quite limited considering "there is so much more implied before, during, and after the operation of algorithms" (Roberge \& Seyfert, 2016, p. 3).

Kitchin (2017) describes algorithms as being "ontogenetic" by nature (p. 16). By this he means that algorithms are "always in a state of becoming" (Kitchin, 2017, p. 18). They are ontogenetic in so far as they are caught in a perpetual negotiation between the various actors making up their existence. They are ceaselessly "edited, revised, deleted and restarted, shared 
with others, passing through multiple iterations stretched out over time and space" (Kitchin, 2017, p. 18). Seaver (2014) similarly points out that algorithms have "hundreds of hands reaching into them, tweaking and tuning, swapping out parts and experimenting with new arrangements" (p. 10). These authors' comments not only highlight the way algorithms operate as networked systems whose power, purpose, and activity are dispersed across actors, but also reveals the way algorithms can be thought of as ongoing processes spread across time.

Bucher (2018) explains that her own approach to studying algorithms is indebted to the perspectives of actor-network theory (Latour, 2005), processual/relational philosophy (Deleuze \& Guattari, 1987; Whitehead, 1978), agential realism (Barad, 2003; 2007), and new materialism (Bennett, et al., 2010; Braidotti, 2006). The work of the scholars mentioned in this section either directly draws from one or more of the aforementioned theories, or presents their own comparable theoretical approaches. What links their ideas together is that they emphasize a relational ontology, and promote a relational materialist approach to analyzing algorithmic networks.

\section{Algorithms and the shaping of cultural tastes and practices}

Scholars such as Beer (2013), Napoli (2014), Morris (2015), Striphas (2015) Urricho (2015) Gillespie (2016), and Cohn (2019) have focused on the role algorithms play as cultural intermediaries or gatekeepers; they question the power algorithms have to sort, rank, and distribute cultural items, and therefore shape our cultural tastes and practices. A recurring concern amongst these scholars is that algorithmic technology is replacing the fundamentally human process of cultural meaning-making, which plays a critical role in the development of social life (Beer, 2013). 
Beer (2013) argues that recommendation algorithms pull our attention to particular cultural products and therefore "exercise the power to shape cultural encounters that then feed into taste" (Beer, 2013, p. 94). Beer worries that algorithmic agency has replaced human judgement, and the tacit knowledge accumulated by cultural experts has been subsumed by massive databases (Beer, 2013). He points out that recommendation algorithms are only some of the most visible aspects of this shift in cultural consumption. They are the surface level actors of what are incredibly complex technical systems that work to "order culture" (Beer, 2013, p. 94). Due to the prevalence of algorithms all around us Beer suggests that we live in a time where culture now "finds us" (Beer, 2013, p. 94).

Morris (2015) examines how Big Data and recommendation algorithms serve in the intermediation of contemporary musical experiences (Morris, 2015). Like Beer, Morris also argues that recommendation algorithms occupy a central position in the circulation of cultural products, and therefore, in the processes of taste-making (Morris, 2015). In order to support this claim Morris looks at the Wreckommender, a recommendation software program that produces "anti-recommendations" based on your favourite musical artists (Morris, 2015, p. 447). Morris argues that the Wreckommender, as well as its more conventional algorithmic counterparts, act as intermediaries between people and culture and that they do so in a similar, but far more effective manner than the likes of talent scouts, radio hosts, critics, marketing managers, etc. (Morris, 2015, p. 451). Morris worries that culture is being reduced to data, and believes that this process is giving way to a new class of services, which he calls infomediaries: "organizational entities that monitor, collect, process and repackage cultural and technical usage data into an informational infrastructure that shapes the presentation and representation of cultural goods" (Morris, 2015, p. 452). 
Building on Galloway's (2006) idea of "algorithmic culture", Striphas (2015) presents a theoretical framework for understanding the current transformation of culture under the "logic of big data and large-scale computation" (p. 396). He defines algorithmic culture as the phenomenon by which "human beings have been delegating the work of culture - the sorting, classifying and hierarchizing of people, places, objects and ideas - to data-intensive computational processes" (Striphas, 2015, p. 396). He uses the \#AmazonFail incident as a backdrop to his investigation. The issue surrounding \#AmazonFail was first brought to attention in 2009 by author Mark R. Probst, who noticed that Amazon had been excluding gay and lesbian themed books from its searches, sales rankings, and best seller lists (Striphas, 2015). Not only does this example highlight some of the social consequences of relying on algorithms, but it also showcases the way in which Amazon has become an "exemplar" of algorithmic culture, in both its successes and its failures (Striphas, 2015, p. 396).

Although significantly less research has been done examining the role algorithms play in the actual production of cultural items, scholars have identified two ways they appear to be shaping this process. On the one hand, algorithmic technology plays a key role in the creative decision-making process, telling producers what content will be successful, with which audience, and why, based on massive user data sets (Napoli, 2014). Bucher (2018) describes this as "data-driven media production" (p. 2). On the other hand, content producers now "orient themselves towards algorithms" by ensuring their content is "algorithmically recognizable" (Gillespie, 2014, pp. 18-19). Due to the increasing need to create algorithmically recognizable content, Striphas (2014) has argued that culture today has not one but two audiences: people and machines. 


\section{The use of Big Data and algorithms in the film and television industry}

Hallinan and Striphas (2016) add depth to the concept of "algorithmic culture" by focusing their attention on Netflix, using the Netflix Prize as their primary case study. The Netflix Prize was a contest launched by Netflix in 2006 that offered \$1 million US dollars to the first team or individual to develop a recommendation system that would improve upon the accuracy of their existing system by $10 \%$ (Striphas \& Hallinan, 2016). Considering the open nature of the contest and surrounding discourse, the Netflix Prize provided an opportunity to scrutinize a major company's use of algorithmic technology. For Hallinan and Striphas, however, it also represented "an effort to reinterpret what culture is - how it is evaluated, by whom, and to what ends" (Hallinan \& Striphas, 2016, p. 119). After examining the discourse surrounding the competition, much of which was taken from the online Netflix Prize forum, the authors argue that there has been a clear semantic shift in how we talk about culture in the digital age. Cultural authority, they argue, has shifted to the realm of "technique and engineering" (Hallinan \& Striphas, 2016, p.119). As a result, cultural authority is no longer in the hands of humans debating great works, but is being attributed to systems of algorithms that, in the case of Netflix, aim to "moderate elements of the cultural field", giving us more of the same and excluding cultural outliers - trapping us in "closed commercial loops" (Hallinan \& Striphas, 2016, p. 122).

Cohn (2019) draws attention to Netflix's more recent push to advertise the use of human handpicking in their content recommendation and labelling system (p. 30). He argues this was evident in Netflix's user interface where "recommendations" switched to "top picks" (Cohn, 2019, p. 30). Cohn argues that this distinction "foregrounds contemporary debates about the value of the labour of AI versus that of humans" and brings attention to what have been mixed consumer reactions towards algorithmic taste-making (Cohn, 2019, p. 30). 
Kelly (2017) examines the increasing use of Big Data in television audience measurement. He interrogates the hype surrounding the use of Big Data in the television industry, parsing out its transformative potential, as well as its often-overlooked limitations (Kelly, 2019). Despite market research always existing within the entertainment industries, Kelly argues it is the "volume, variety and velocity" of Big Data that distinguishes it from past methods (Kelly, 2019, p. 115). Kelly borrows Braun's term, "transparent intermediary" to describe the invisible technological infrastructure - now made up of Big Data and algorithms that underpins and shapes "every facet of television culture" (Kelly, 2019, p. 117). He also argues that Big Data privileges real-time analysis especially in the form of social media metrics, which is in-turn leading to the "eventization" of mainstream television (Kelly, 2019, p. 122). For this reason, Kelly suspects that networks, producers, and advertisers will increasingly look to invest in content that generates high levels of social media activity (Kelly, 2019, p. 122).

Burroughs (2019) attempts to uncover the current "industry lore" surrounding Netflix and the streaming industry. He argues that Netflix actively promotes public discourse around algorithmic technology in order to support their brand, positioning their algorithm "as the solution to the complications of fragmentation for media industries in an era of globalization" (Burroughs, 2019, p. 10). He argues that in their consistent claim that their recommendation system will guarantee consumers the content they want, Netflix "discursively situates the audience through the promise of the black-boxed algorithm" (Burroughs, 2019, p. 5). Burroughs sees the discourse surrounding the use of algorithms in the streaming industry as being largely promoted by the companies themselves, providing an example of what he calls "streaming lore" (Burroughs, 2019, p. 2).

Ultimately, there appears to be less scholarly work looking at the role Big Data and algorithmic technology play in the development and production of film and television. However, 
when it is discussed there is one commonly cited example appearing throughout several related articles: Netflix's strategic use of user data to develop and produce their original series House of Cards (see, e.g., Burroughs, 2019; Hallinan \& Striphas, 2016; Smith \& Telang, 2016). Although this example was widely publicized and frequently cited in academic literature, it appears to be one of the only examples and case studies that has been explored. 


\section{Methodology}

This paper has adopted Bucher's $(2016,2018)$ methodological approach of technography, which aims to describe and observe the "complex ways in which software intersects with sociality in a way that is similar to anthropological methods of ethnography" (p. 86). Technographic inquiry is less concerned with revealing the technical inner workings of software (in this case, locating the exact formula of an algorithm and the data structures it operates on); rather, the technographer asks how meaning is materialized in technology and questions the impact software itself has on society (Bucher, 2016, p. 87). Adopting a technographic approach to study algorithms is to examine the complex interplay between the variety of actors involved in an algorithm's becoming and to assess how new forms of social and cultural meaning develop as a result of these constitutive entanglements. Technography, like ethnography, is a flexible methodology: “just as ethnographers assemble notes, interviews, and observations into a new account of a cultural phenomenon, technographers build their account of algorithms by assembling information from different sources" (Bucher, 2016, p. 87). Rooted in a relational materialist perspective, technography allows for the researcher to choose from a broad range of methods, some more traditional (e.g. content analysis) and others more abstract or experimental (e.g. reverse-engineering). In addition, it welcomes entirely new and innovative tactics. No matter the specific method, what is crucial to the technographer is finding unique ways to make software “offer a description of itself” and/or to locate instances and areas where algorithms make themselves and their meanings visible. Accordingly, the technographic approach is particularly interested in the way algorithms operate discursively: how they are being communicated, discussed, promoted, challenged, and imagined in the public domain (Bucher, 2016). 


\section{Research methods}

In order to explore the complex and multilayered role algorithms play in the production, distribution, and consumption of film and television, I have used two specific technographic methods outlined by Bucher (2018): the employment of a phenomenological perspective and the use of reverse engineering.

\section{Phenomenological Perspective}

Bucher (2016) stresses that when it comes to studying algorithms, social science and humanities researchers should "not expect the solution to be inside the black box" (p. 88). Therefore, another tactic she proposes is to adopt a phenomenological perspective when approaching algorithms, which entails being "attentive to the ways in which social actors develop more or less reflexive relationships to the systems they are using and how those encounters, in turn, shape online experience" (Bucher, 2018, p. 61). Employing this tactic requires researchers to shift their focus to the ways in which life "gains expression through encounters with algorithms" (Bucher, 2018, p. 61). This tactic is especially appropriate for examining the ways algorithms operate discursively and/or reveal themselves in public discourse. I adopt a phenomenological perspective in order to analyze the evolving role Big Data and algorithmic technology are having on the creative process as it pertains to the development stage of film and television production.

\section{Reverse Engineering}

Bucher (2016) posits that researchers must ensure they "do not fear the black box" and instead tackle the discoverable aspects of any algorithm (p. 85). One of the methods she suggests for accomplishing this is through reverse engineering; a process that seeks to extract knowledge about the operational logics of algorithms through "speculative experimentation and playing 
around" (Bucher, 2018, p. 60). Because technography is concerned with how algorithms form new social and cultural meanings, engaging with them directly through the platforms they are embedded in - and doing so in a calculated manner that allows for the careful observation of their procedural outcomes - can be particularly revealing. This method has been successfully implemented by a handful of researchers in the past (see e.g., Bucher 2012; Diakoplous, 2014). It is also worth noting that Sandvig et al. (2014) promote a similar method that they call the algorithmic audit.

For the purpose of this paper I have 'reverse engineered' the Netflix Recommender System, by setting up three Netflix user accounts with three distinct taste profiles. Every day for two weeks (May 24 $4^{\text {th }}, 2020$ to June $7^{\text {th }}, 2020$ ) I selected one new film or television show from the homepage screen (which is entirely algorithmically generated) according to each profile's "tastes". I recorded the titles of the films and shows selected, as well as the altgenre Netflix attributed them to. Much like Bucher (2012), I simply tracked any changes by taking screenshots of the homepage every day, before a selection of a film or show had been made. I interpreted the results by comparing the different homepages for each taste profile. Not only did this reveal differences in what films and shows were being algorithmically recommended, but also how they were organized and ranked within Netflix's interface. For example, Netflix deploys three layers of algorithmic personalization, all of which impact user navigation: content is organized into custom "rows" (e.g. "Award-Winning Dramas") suited to your profile preferences, which are then ordered based on what you are most likely to engage with; the titles in those rows are algorithmically chosen; and the appearance of those titles within the rows is also ranked and ordered (Netflix, n.a).

I would also like to note here that my inspiration and general approach for this experiment was in keeping with the ideas and guiding principles outlined by Latour's (2005) 
actor-network theory (ANT). In particular, his emphasis on the prioritization of description-asexplanation, and the suggestion to "follow" both human and non-human actors in hopes to let the explained become the explainer. 


\section{The Streaming Wars}

On Wednesday, November $12^{\text {th }}, 2019$, Disney released their streaming service Disney+ throughout North America. Within twenty-four hours the service had gained ten million subscribers, greatly surpassing analyst predictions (Barnes, 2020). By Wednesday’s close, Disney's stock had risen $7.35 \%$, adding $\$ 13$ billion to its market cap. Meanwhile, Netflix's shares had dropped 3.1\% (Feiner, 2020). Although Netflix, Amazon, and Hulu had been battling over consumer subscriptions for well over a decade, it was Disney's impressive launch into the market that truly ignited the "Streaming Wars", a sensational title used to describe the escalating competition between OTT video streaming services. Flash-forward to Spring of 2020 and the streaming market is now crowded by some of the most valuable companies on the S\&P $500-$ Apple, Amazon, AT\&T, Disney, Comcast, and Netflix - all of whom are pouring billions of dollars into OTT video content and solutions (Spangler, 2020).

The Streaming Wars represent a particularly important moment for legacy media companies, namely Disney, WarnerMedia, and NBC Universal, who for years had struggled to adapt to the "distribution revolution" happening within the film and television industry (Sutter, 2014). The distribution revolution arrived thanks to a new media ecology that had emerged as a

result of a "bandwidth explosion, more powerful laptops, personal mobile technologies, and the advent of cloud computing” explains Curtin et al. (2014, p. 27). This ecology was situated around constant connectivity, user interaction, and data collection; and was established well outside the pervasive reach of traditional media conglomerates that for nearly eighty years had relied on oligopolistic industrial structures and business models of vertical integration to exert massive control over every facet of the film and television industry. CEOs of these companies were now faced with an "unusual reversal for the media industries [where] business models must now be crafted in response to consumer behavior rather than as a means of engineering or 
controlling that behavior" (Holt, Steirer, \& Petruska, 2016, p. 342). The passive film and television consumer of the $20^{\text {th }}$ century had evolved into the networked viewer, an active subject in the processes of production, who expected content to be delivered instantaneously at their choosing, across multiple platforms, and curated to their personal accounts. In the face of these shifting dynamics, the strategies of major studios and networks often appeared reactionary, rigid, and late to the game (Barnes, 2012). Bogged down by their own bureaucracy, industrial structures, and old school leadership "many powerful firms have stumbled and lost ground on markets they used to dominate" explain Smith and Telang (2016, p. 3). Meanwhile, the "digital native" streamers - Netflix, Amazon, and Hulu - saw a business opportunity presented by this tumultuous transitionary period to rebuild the film and television company from the ground-up. Cunningham (2013) refers to Netflix, Amazon, and Hulu as the "disruptive innovators" in reference to their transformative impact on existing industry practices and power structures (Cunningham 2013, as cited by Crisp, 2015, p. 60). These companies significantly altered film and television practices in ways that now define the foundational elements of the Streaming Wars. Some of these important changes include the following: 1) an innovative direct-toconsumer distribution model that operates over the Internet, free from the control and structures of wired cable networks and major film studios (hence the name "over-the-top"); 2) the use of Big Data and algorithmic technology for both green lighting and curating content; 3) less restrictive approaches to developing content, and therefore greater levels of freedom for writers and creators (they had bypassed the constraints of broadcast advertising breaks, as well as standardized season lengths and program runtimes); and 4) new release strategies, and in turn, novel forms of consumption (e.g. binge-watching); and even new cultural practices from multiscreening to Netflix and chilling. 
After years of hesitancy, as well as several cursory attempts at entering the streaming market (e.g. Disney Anywhere, Ultraviolet, and Comcast's Xfinity), Disney, WarnerMedia, and NBCUniversal have finally embraced the "distribution revolution" head-on. They have, in part, abandoned the struggle to protect their traditional business models and are now betting large on the success of their own streaming services. Disney spent $\$ 71$ billion dollars acquiring $21^{\text {st }}$ Century Fox, which was in large part a decision made to eliminate streaming competition and build-up Disney +'s content library (Jarvey, 2019). AT\&T has devoted $\$ 4$ billion dollars to developing HBO Max over the next three years (Bursztynsky, 2020), and Comcast vows to spend \$2 billion dollars on NBCUniversal's Peacock within its first two years of operation (Spangler, 2020). Just as Hollywood had been ruled by the 'Big Five' film studios for nearly a century, five major players have also begun to emerge during the early stages of the Streaming Wars, whom I will be referring to as the Big Five Streamers: Netflix, Amazon Prime, Disney+, Hulu (which is owned by Disney), and HBO Max. ${ }^{1}$ Battling for a sixth position is Apple TV+, Peacock, CBS All Access (owned by CBSViacom) and to a lesser extent Jeffrey Katzenberg's experimental mobile-only streamer Quibi.

As for the other major studio conglomerates - ViacomCBS, Sony Pictures, and Lionsgate - they will be taking what Littleton and Low (2019) called the "arms-dealer approach" to the Streaming Wars: "This evolution has bifurcated the largest studio conglomerates into two types of companies: those marshaling content armies (Disney, AT\&T and Comcast) and those who aim to be content arms dealers (ViacomCBS, Sony Pictures, Lionsgate)." Rather than developing their own OTT services, these companies will primarily rely on licensing their own content to the highest bidding streamer, which includes their extensive film and television archives (Littleton \& Low, 2019). However, it is important to note that CBSViacom has taken a

\footnotetext{
${ }^{1}$ I based this list according to IndieWire's March 1st “Top 5 Power Ranking” (Travers, 2020).
} 
multi-tiered approach, licensing content to other streamers, while still offering their own OTT streaming services: CBS All Access and Pluto TV. Key to their strategy is reintroducing traditional advertising revenues into the streaming space, a tactic also being adopted by NBCUniversal with Peacock (Little \& Low, 2019).

In addition to these major corporations, there has also been a proliferation of niche streaming services. In October of 2019, research firm Parks Associates announced that there were 271 streaming services available in the United States (Barnes, 2019). Some popular examples include, the Criterion Channel targeted at film buffs, Shudder for horror lovers, and Crunchy Roll for fans of anime. Adding to the incessant fragmentation of the industry, there is a parallel battle being waged between streamers offering live television specifically. These include Youtube TV, Hulu Live, DirectTV, Sling, Philo and again, Peacock, CBS All Access and Pluto TV. All of these services abide by traditional broadcast schedules offering bundles of top cable channels, with some even offering local channels based on user geolocation. These services are quite literally transferring the cable viewing experience to the Internet, though they provide typically sleeker and more intuitive interfaces, as well as additional functions such as DVR. This has given way to the 'cord cutting' phenomena, where customers are increasingly abandoning their subscriptions to traditional cable and satellite packages, and opting for cheaper OTT options. According to research by Deloitte (2020), $80 \%$ of all U.S. consumer households subscribe to at least one OTT streaming service, up 7\% from their pre-COVID survey. And in 2019, all U.S. cable providers saw a massive drop in subscribers, losing a combined six million customers over the course of the year, a 7\% year-over-year decline (Spangler, 2020b).

Further complicating matters, many of the aforementioned services follow different monetization models, with the most common being subscription video on demand (SVOD), adsupported video on demand (AVOD), and transactional video on demand (TVOD). Several 
services even combine these models. For example, Peacock offers three price tiers: a free adsupported version with limited content; a $\$ 4.99$ US subscription with all available content and advertising; and a \$9.99 US premium subscription with all content and no advertising. Similarly, while YouTube is first and foremost a free AVOD service for watching online video, the company also offers a pay subscription version of the service for premium content (YouTube Premium), a built-in TVOD service that offers thousands of film and television titles for onetime purchase or rent, and a separate live streaming TV service (Youtube TV). Strangely, YouTube has received far less media attention in regards to the Streaming Wars narrative, despite the fact that people are consuming a staggering 1 billion hours of content through the service every day (Youtube, 2017).

In addition, several of the new streaming services can be purchased in bundles, if offered by the same parent company. For example, Disney offers multiple bundling options for Disney+, with its most popular option being the "Disney Bundle" which includes Disney+, Hulu, and ESPN+. Meanwhile, AT\&T, Comcast, and CBSViacom are giving away their respective streaming services - or at least certain tiers of them - for free to customers already subscribed to their various video, internet, and mobile plans; Verizon does the same for Disney+ (Bursztynsky, 2020; Hayes, 2020; Kidwell, 2020).

Therefore, a crucial macro-level trend to consider is the ways these companies are using streaming services as tools to entice consumers to buy into larger media ecosystems. Matthew Ball (2020), industry analyst and former Global Head of Strategy for Amazon Studios, argues that the Streaming Wars are a by-product of a broader "ecosystem war" being fought between the largest technology and media companies in the world. Due to this, streaming services and the content they offer are becoming increasingly entangled in a much more complex and opaque valuation process. This is particularly notable in the case of Amazon and Apple, two non- 
traditional media companies that have entered the streaming market in full-force. Amazon, an eretailer and cloud service provider, positions its video streaming service, Amazon Prime Video as being "free" with a subscription to Amazon Prime. So, despite the company spending an estimated \$5 billion dollars a year on content for Prime Video (Ball, 2020), the service is ultimately only one feature within the Amazon ecosystem, where it operates synergistically to funnel consumers into the broader e-retailer experience; paying for a sustained membership to Amazon Prime year after year becomes significantly more reasonable for consumers if they are expecting new seasons of their favourite show to be released annually on Prime Video. CEO of Amazon, Jeff Bezos, has been incredibly vocal about the synergistic approach to developing Prime Video, saying:

When we win a Golden Globe, it helps us sell more shoes. And it does that in a very direct way. Because if you look at Prime members, they buy more on Amazon than nonPrime members, and one of the reasons they do that is once they pay their annual fee, they're looking around to see, 'How can I get more value out of the program?' And so they look across more categories - they shop more. (McAlone, 2020)

This also explains why Apple is offering Apple TV+ at the aggressively low price point of \$4.99 US a month, in addition to giving away the service for free for a year to millions of Apple customers who have purchased an apple device, computer, or even student subscription to Apple Music (Alexander, 2020). According to Ball (2019) Apple TV+, just like Apple Music, will operate as a structurally unprofitable service that intentionally looks to generate revenues elsewhere in the Apple ecosystem. Just as Prime Video helps Bezos sell shoes, Apple TV+ should, in theory, lead to greater sales of iPhones. Because of this, the idea that the Streaming Wars are being fought just for total subscriber numbers, or even max profits, is a common misconception. Really, as Ball argues, these companies are battling to monopolize consumer 
spend, data, and more abstractly, free time (the more time spent on one service means the more time spent within one company's ecosystem versus their competitors). This explains what can appear to be trivial differences between these services business models, which in reality reflect their varying goals, as Ball (2019) explains:

Some of them charge, some don't; some will generate direct profits, some won't; some aspire to maximize view time, others just want a part of it. However, they all share a common business strategy: they're not focused on the video TAM ("total addressable market" value). Instead, they're being used to access other, more lucrative ones.

Sometimes these TAMs are based around high-margin consumer devices, other times it's trillions of dollars in retail spend, hundreds of billions in wireless service revenue, or terabytes of consumer data. (Ball, 2019)

Ultimately, while OTT streaming services have revolutionized distribution, it has become clear that they are far more than just distributors. More appropriately they should be conceived of as platforms that exist and operate at the intersection of a myriad of industrial, economic, cultural, social, technological, and political developments. Here, I borrow Van Dijck and Poell's (2016) definition of platforms as "online sites that facilitate and organize data streams, economic interactions, and social exchanges between users" (p. 2). As platforms, services like Netflix, Hulu, Disney+ and HBO Max, are the ultimate products of convergence. They are at one time distributors, as well producers of content, entertainment providers, as well data miners; they combine various technologies and forms of media, as well blend numerous industries under one company name (Buck \& Plothe, 2019). Furthermore, many of these services now operate within much larger consumer ecosystems where their value is often widely dispersed, and therefore difficult to measure from a third-party perspective. As a result, the cultural practices associated with film and television are also becoming increasingly complex and networked (more on this in 
the following sections). These are the new dynamics defining the Streaming Wars, which itself signifies a new era in film and television history. And while there are many elements to dissect in regards to the emerging OTT film and television landscape, a good place to start is by first considering the technologies it has been built upon, as the complexity of the Streaming Wars only mirrors that of the algorithmic technologies defining its parameters. In the following section I will explore the way Big Data and algorithmic technology are impacting the film and television production process. I will focus my analysis on emerging third-party firms that aim to algorithmically automate key creative practices undertaken during the development stage of production. 


\section{Data-Driven Creativity and Networked Cultural Production}

Producing compelling and profitable content is the key to a film or television studio's success, but when it comes to explaining what generates that success, the answer has been the same for nearly 40 years: "nobody knows anything." This mantra comes from legendary screenwriter William Goldman who famously wrote, "not a single person in the entire motion picture field knows for a certainty what's going to work" (Goldman, 1983). Thurau and Houston (2019) argue that Goldman's words have formed "the economic foundation of decision making for several generations of entertainment managers" in both film and television (p. 4). Implied by the mantra is the importance of managerial intuition and 'gut instinct' when it comes to picking the right script, choosing a new star, or developing a hit-show, which is why its use and popularity within the industry remains primarily idealistic. Large studios have never ceased investing millions of dollars into market research and analysis in order to guide the development of future content. Even at the time Goldman was writing his soon-to-be industry saying, there were extensive efforts being made by studio executives to make certain the success of their films and shows via rudimentary data collection and market research. This included box office ticket counting and the analysis of television ratings, as well as the deployment of consumer focus groups, test pilots, polling, and surveys (Simon \& Schroeder, 2020). In fact, Ien Ang (2006) has argued that it is audience measurement and "ratings discourse" that have allowed for the institutional discursive construction of "audience-as-market," which has defined the historical development of television and framed both industry and consumer perspectives since the 1970s, following the implementation of setmeter's and family diaries by ratings firms like Nielsen. Ang argues that:

through the symbolic world created by ratings, a world inhabited not by actual audiences but by a discursively constructed 'television audience', the industry has armed itself with 
a guiding principle for solving the multiple dilemmas, problems and disputes which the gigantic enterprise of commercial broadcasting entails. (p. 42)

When cable television and the VCR were introduced in the 1980s, they were incredibly disruptive technologies from an institutional perspective due to their ability to obscure ratings measurement: viewers could now 'surf' between hundreds of cable channels and use their VCR's to record and watch shows independent of broadcast schedules, making diaries and setmeter's ineffective measurement tools (Ang, 2006). In response, the ratings industry had quickly developed the "people meter," an electronic monitoring device that could record individual viewing habits through a portable remote (Ang, 1991). The "people meter" introduced a technique for measuring the "unknown minutiae in aggregated viewing habits" and therefore, represented a discursive shift at the institutional level towards a new emphasis on monitoring 'viewing behaviour,' one which would presage the dominant discourse surrounding Big Data and OTT streamers today (Ang, 2006, p. 64). As for the movie business, audience measurement had historically been more straightforward. Richard Maraschi, global leader of analytics at IBM, explains that Hollywood had for decades relied only on "quad segment" consumer analysis, which consisted of four basic characteristics: male versus female, and over or under 25 years of age (The Atlantic, 2020).

Of course, these tactics were only so reliable, as well as relatively low-resolution in comparison to the rich and granular level of consumer data analysis enabled by the Internet, which has made the calculated development and production of content more affordable, sophisticated, and pervasive. In today's competitive streaming landscape, the leveraging of large-scale data analytics has become a defining feature of film and television production. The OTT streaming business model is built on a two-way relationship between consumers and producers, in which content flows out and algorithmically readable consumer data flows in. 
Raustiala and Sprigman (2019) highlight some of the diverse and detailed aspects of user behaviour being collected and put to strategic use by Netflix:

Some parameters that Netflix tracks include, but are likely not limited to, pause/rewind/fast-forward behavior; day of the week; date of viewing; time of viewing; zip code; preferred devices; completion rate; user ratings; user search behavior; and browsing and scrolling behavior. (p. 1587)

In spite of the Goldman mantra, it appears that OTT streamers like Netflix "know" quite a lot about their users and can determine what content may "work" to an exceptionally accurate degree. Their data-centric business models have changed the way content investment and production decisions are being made, giving rise to what many are calling "data-driven programming" (Bucher, 2018), or what Lazar (2015) describes as "arts analytics". A widely cited example of such data-driven production is Netflix's development of their hit show House of Cards. Netflix was so confident in their own data analytics that after receiving the pitch for the show they committed $\$ 100$ million dollars to the licensing of two seasons without producing a pilot, completely bypassing the traditional and expensive broadcast process of using pilots to gauge audience interest (Smith \& Telang, 2016). In an interview discussing the development of the show Ted Serrandos, Chief Content Officer at Netflix, said "we can draw real data pools of people who love Kevin Spacey, David Fincher movies, the original House of Cards, political thrillers, and on and on. You wind up with a very predictable pool of viewers" (Curtin, et al., 2014, p. 137). Similarly, it was data that led Netflix to sign two massive four-film deals with Adam Sandler, despite his recent starring in a series of box office flops, which were notoriously panned by film critics; the data showed that Sandler was the most watched actor on the platform finding particular resonance in Latin America, making him a bankable star regardless of his then dwindling reputation (Greenberg, 2015; Friedman, 2017). 
While Netflix's incorporation of Big Data in the production process had given them an initial competitive advantage, this kind of "data-driven programming" is likely to become quite commonplace as the Streaming Wars continue to unfold, and considering the history of audience measurement it is not necessarily all that new. This is why I wish to explore Raustiala and Sprigman's (2019) broader concept of "data-driven creativity”, as it more aptly describes the next novel change facing the industry in regards to the impact Big Data and algorithmic technology are having on the creative process. Data-driven creativity describes not only the way data is used retroactively to guide the development of future content or how it is leveraged for marketing and promotion (though it includes these elements), but also the phenomenon by which Big Data, in combination with algorithmic technologies - namely, machine learning and AIplays an increasingly direct role in the creation of content itself (Raustiala \& Sprigman, 2019). In the case of film and television, data-driven creativity is at its most palpable when it is being deployed during the development stage of production specifically.

Development is the first stage of a film or television production and includes all activities leading up to the "greenlight" decision that a movie or show will be made (Behren et al., 2020). According to Behren et al. (2020) this stage includes "many interrelated and iterative processes, including ideation, script development, casting, audience analysis, demand forecasting, and financing" (p. 6). Essentially, the development stage is where new content ideas are sought out, brainstormed, refined, written, budgeted, and pitched by a small group of either in-house or independent creatives and executives. Despite the emphasis on creativity and collaboration at this stage of the production cycle, innovations in data analytics, machine learning, and AI are being increasingly utilized for development purposes. Some of the most noteworthy examples of data-driven creativity creeping into this highly imaginative stage of production come from emerging third-party firms such as Vault AI, Scriptbook, and Cinelytic. These firms, and others 
like them, are using Big Data and algorithms to assist or replace the fundamentally creative practices found at every level of the film and television production cycle. However, it is the tools and services they offer for the development of film and television that are particularly illustrative of data-driven creativity and its future potential.

The remainder of this section of my MRP will examine these emerging firms and their innovative technologies, by highlighting three key development practices that they are currently disrupting: ideation, script development, and casting. Like Netflix and Amazon, the exact systems of algorithms being used by these companies are proprietary, and therefore remain unknown. But because they operate outside of the control of large studios and major streamers, often marketing themselves as platforms available for public use, these specialist firms provide a valuable window for research into the current role Big data and algorithms play in the film and television production process. It is also worth noting that while I have organized these companies according to specific development practices, the majority of them provide a wide variety of analytic services crossing all stages of production (i.e. development, pre-production, principal photography, post-production, and distribution).

\section{Ideation}

Ideation describes the creative process of generating, developing, and communicating new ideas (Britton, 2017). A new wave of companies utilizing large-scale data analytics and AI-led content analysis are offering producers and filmmakers assistance with the ideation process specifically. Broadly conceived, their objective is to provide unbiased, data-driven recommendations in regards to what kind of stories to tell, as well as how to tell them. Vault AI is an Israeli based technology company using machine learning and predictive analytics to "understand how stories can reach their maximum potential" (Vault AI, 2020). The company aims to integrate science 
and data measurement into the ideation process, replacing old-school methods such as surveying and focus groups, while mitigating the risks associated with pure intuitive decision-making. Vault AI's "DeepAudience Analysis," intends to predict a film or television show's future audience and potential success based on the "DNA" of the creative, as it stands at the development stage (Vault AI, 2020). The company has trained their AI system on "billions of audience and story data points" derived from over 30,000 film or television titles. They claim that their AI system can accurately analyze any "script, outline, finished cut, and trailer" and provide actionable insights months or even years in advance of release (Vault AI, 2020). The data-driven recommendations offered by Vault AI range from how to improve a show's concept and story elements such as theme, characters, and dialogue, to suggested release sizes and key geography breakdowns for blockbuster films. According to the company's website, their AI system can even determine whether a series is optimized for basic cable, premium cable, broadcast television, or OTT, as well as predict how many seasons the series would likely run for (Vault AI, 2020).

In a blog post written by Vault AI's CEO David Stiff, he provides a breakdown of their analysis and predicted success of Warner Brothers hit film Aquaman (2018), which grossed more than 1 billion dollars worldwide, despite low expectations from industry analysts (Thompson, 2020). In order to assess the financial potential of Aquaman, Vault AI analyzed “over 100,000 story elements" from the film's first released trailer, which came 22 weeks before its premiere. In doing so, their AI system was able to "extract the core story DNA" of Aquaman and assess how the film might resonate with domestic and international audiences (Stiff, 2020). Based on key story elements labelled, "superhero origin story", "Jason Momoa”, "family / mentor relationships", and "blend of the futuristic and mythical", in combination with specific "beats" (moments of action) like special FX "surface battles", the Vault AI system was able to 
predict the following domestic numbers: $\$ 74$ million opening weekend (\$67.8 million actual); exit polls to be M 53\% / F 47\% (M55\% / F45\% actual exit polls); and Rotten Tomatoes Audience Score to be 77\% (79\% actual score) (Stiff, 2020). Their AI system even found interesting differences between how international markets reacted to the trailer. For example, Stiff (2019) explains how their trailer analysis revealed that the "Asian market generally lacked interest around the comedic beats, especially the wisecracks and showed stronger interest in the more serious, explosive action-sequences."

While Vault AI's film trailer analysis is certainly innovative, there is a tendency to assume that this kind of technology is so new that it remains sparsely used within the industry; however, there is evidence of major studios employing this exact method. In 2018, data scientists working at $20^{\text {th }}$ Century Fox (now owned by Disney), partnered with Google's Advanced Solutions Lab to develop a machine-learning and advanced computer vision software tool named Merlin Video. The tool is used to analyze and label thousands of movie trailer elements, as well as process robust sets of granular customer data in order to predict a film's future audience, based solely on its trailer (Campo-Rembado \& Oakley, 2018). According to Campo-Rembado and Oakley (2018), the two data scientists leading the project, Merlin Video has become a routine consideration "for important business decisions" providing $20^{\text {th }}$ Century Fox with "one of their most objective, data-driven, and effective barometers to evaluate the tone of a movie, its affinity with core and stretch audiences, and its potential financial performance."

While the specifics of Vault AI's technology remain elusive, $20^{\text {th }}$ Century Fox and Google have published a paper regarding the development of Merlin, allowing for greater public insights in regards to the technical aspects of the system. Unlike the more popular approach to video analysis, which breaks down a video into individual frames using "trained image classification deep architecture", Merlin uses a "temporal-aware" model that takes into 
consideration the temporal dynamics of a movie trailer in order to capture salient elements only expressed across sequences of frames (Hsieh et al., 2018). It then labels these elements and compares them to large data sets of other film trailers, as well as customer attendance records in order to make predictions via collaborative filtering.

\section{Script Development}

Arguably the most important element during the development stage, which is inherently tied to ideation, is script writing, revising, and buying. Script analysis services, which rely on largescale data analytics and natural language processing (NLP) are currently emerging on the market, offering producers and screenwriters a new way to value their scripts based on AI-led insights $^{2}$. Script analysis can be utilized by producers and screenwriters in two interrelated ways: as a guide for greenlighting scripts according to box office predictions, and as a practice for improving a script's story elements.

While Vault AI, and several other firms (e.g. Epagogix and Storyfit) offer some form of script analysis to complement their other services, the current leader in this field is Scriptbook, a Belgium-based artificial intelligence company focusing solely on AI-led script analysis. The company employs machine learning and NLP to assess a script's individual story elements, as well as its overall style and structure, which, taken together, make up what the company calls a "script fingerprint" (Scriptbook, 2018). Similar to trailer analysis, their AI system compares a script fingerprint to thousands of other deconstructed film scripts stored within their database, which date back to 1970 (Buder, 2017). The system then produces a series of predictions regarding that script's potential for commercial and critical success.

\footnotetext{
${ }^{2}$ NLP is a branch of artificial intelligence that aims to read and understand the semantic relations embedded in human language (Behrens et al., 2020).
} 
Scriptbook markets itself as an easy-to-use service, in which users upload a script and within 24 hours receive an in-depth analysis of their story, as well as specific audience predictions and financial forecastings. These items are packaged together in a report that includes, but is not limited to, the following items: individual scene analyses, character likeability analyses, character emotion summaries, predicted genre and MPPAA rating, predicted IMBD and Rotten Tomatoes score, predicted box-office demographic, suggested marketing position, predicted production budget, predicted US and international box-office numbers, and predicted worldwide ROI (Scriptbook, n.d.).

In addition to script analysis, there have also been developments in generative AI systems that are capable of writing film scripts from scratch, with no human intervention. Scriptbook is currently developing one of these systems and in a recent blog post discussed how it required them to "reverse" the processes of their script analysis tool: "We created a generative engine by plugging parameters from our existing AI for script analysis, into a language model resulting in improved writing capabilities and story consistency" (Scriptbook, 2018). The company explicitly states that they do not intend to create a system capable of replacing the screenwriter, but rather hope to develop a "helpful co-creator for producing written content" (Scriptbook, 2018). While Scriptbook's generative engine currently remains in the development stage, there have been other preliminary attempts at AI-led scriptwriting within the industry. The short science-fiction film "Sunspring" which premiered at the Sci-Fi London film festival in 2016 as a contestant in the "48 Hour Film Challenge", was entirely written by AI. The film was a product of director Oscar Sharp, and Ross Godwin, a creative technologist at Google. Sharp was interested in making a film "out of random parts" and had long dreamt of collaborating with AI on a screenplay (Newitz, 2016). He teamed up with Goodwin who developed an AI system capable of reading and writing scripts, which named itself Benjamin. The specific type of AI 
comprising Benjamin is referred to as a long-short-term memory (LSTM) recurrent neural network, which is often used for text recognition (Newitz, 2016). Before requesting Benjamin to write its own screenplay, Goodwin had trained the system on dozens of science-fiction scripts from popular film and television shows such as Ghostbusters, Interstellar, and X-Files (Hal 90210, 2016). Sharp directed the film, following Benjamin's stage directions as precisely as possible and had the actors interpret the emotional tone of the mostly haphazard dialogue (Newitz, 2016). While "Sunspring" ended being largely incoherent, the film was well-received due to its experimental nature, providing audiences with a strange glimpse into the future potential of AI-led creativity.

\section{Casting}

Casting suitable talent for a film or television show is crucial to ensuring the commercial and critical success of a given project. Just like ideation and script development, the highly intuitive casting process is being redefined by the promises of Big Data and algorithmic technology. In 2018, WarnerMedia signed a deal with start-up Cinelytic, a SaaS (software as a service) based platform that combines AI, data analytics, and cloud-based technologies in order to assist studios and producers with the development of their films and shows (Business Wire, 2020). While the company offers predictive analytics and management tools "throughout the content value chain", they are particularly recognized within the industry for their innovative talent analytics service, which goes far beyond box-office measurements and the individual tracking of directors and actors (Business Wire, 2020). According to Behrens et al. (2020), Cinelytic "utilizes data about directors, actors, writers, and producers, in combination with historical box office [data], budget, and social media metrics, to derive proprietary talent scores", which they organize and offer on a user-friendly web-based platform (p. 13). The talent score of a specific actor, director, 
screenwriter, etc. denotes their estimated economic impact on a project, which fluctuates according to dozens of variables. As James Vincent (2020), a journalist writing for The Verge put it, "Cinelytic's software lets customers play fantasy football with films"; the platform allows producers to input talent and tweak individual project elements such as genre, budget, and release territory in order to automate varying predictions about its potential success. For example, if a studio was planning to produce a comic book-based action film with a female lead, they could use the system to first assess the mostly highly ranked actresses according to their talent score and then swap them in and out to compare the film's overall performance.

A particularly innovative tactic being utilized by Cinelytic is their collection and analysis of legal and illegal peer-to-peer sharing. According to journalist Chris Tribbey (2020), Cinelytic records up to 100 million content downloads and streaming transactions daily, which for the company "serves as a proxy for digital OTT demand across all platforms". Therefore, in addition to collecting box office numbers and mining word-of-mouth (WOM) data from social media sites, the company is able to track the real-time performance of films, shows, and their respective stars by monitoring high traffic piracy networks. In doing so, Cinelytic joins companies like Parrot Analytics and TV Time, in attempting to fill the massive 'ratings void' created by OTT streamers like Netflix and Amazon, who do not publicize the specific viewing numbers of their films and shows.

\section{Retiring the Goldman Mantra?}

Big data and algorithmic technology are being increasingly integrated into the film and television production cycle, reshaping the competitive landscape and redefining the creative process. Data-driven creativity is a concept used to describe the way Big Data and algorithms are impacting the creation of content itself, not just influencing the ways content is marketed and 
sold. I have argued that today, data-driven creativity in the film and television industry is manifested most acutely, and presents itself most tangibly from a research perspective, during the development stage of production. This is particularly apparent considering the emergence of third-party firms like Vault AI, Scriptbook, and Cinelytic, who are leveraging Big Data and AIled innovations in order to automate the highly creative practices of ideation, script development, and casting.

In addition to Vault AI, Scriptbook, and Cinelytic there are several other successful thirdparty firms offering Big Data and AI-led development solutions. These include: Storyfit, a startup based out of Austin, Texas, that analyzes customer viewing history, web searches, and user engagement to assess what kinds of stories the public currently finds most compelling (Behrens et al., 2020); Movio, a New-Zealand based technology company that boasts a massive collection of global "moviegoer data" that includes over 750 million behavioural and transactional records, through which the company claims to "know what your movie goers want to see before they do" (Businesswire, 2019; Movio, n.d); and Epagogix, which has been referred to as one of the leading "neural network consultancies" in Hollywood (Collin, 2019).

While these emerging analytic services are often framed only as additional options in a producer's toolkit (Simon \& Schroeder, 2020), I believe their proliferation in the market is representative of the beginnings of a new era of data-driven creativity. Data and algorithms have already had a profound influence on the distribution of film and television, as evidenced by the Streaming Wars, and I believe they will now continue to push boundaries and alter practices at the production level. From a macro perspective, the entire industry is undergoing a re-ordering due to the competitive necessity of Big Data ownership for both production and distribution processes. For example, AT\&T and WarnerMedia fought as hard as they did to merge in 2018 because both companies recognized the need to expand their access to consumer data in order to 
"reduce the mismatch between creative work and consumer tastes" (Raustiala \& Sprigman, 2019, p. 1605). On a micro level, the practical use of a scriptwriting AI like Benjamin may still seem far-fetched, but the utilizing of AI-led script analysis is not: Smith and Telang (2018) remind us that "if you've watched a reasonable number of studio pictures in the past 10 years, you've almost certainly encountered at least one that has undergone an algorithmic rewrite." Furthermore, we can safely assume that the tools and analytic services being offered by these speciality firms - including the AI-led content analysis of scripts, trailers, audience reviews, posters, and ads, as well as robust talent analytics, storytelling and visualization tools, and even P2P data mining - are also being deployed in-house by the major streamers, likely with greater financial backing and a higher degree of technical sophistication considering their Silicon Valley roots.

As the industry enters a new era of data-driven creativity, now is an appropriate moment to retire the Goldman mantra. To claim that Hollywood is an industry where "nobody knows anything" is ludicrous in the face of OTT streamers capable of mining and analyzing user data at the granular level of interface scrolling behaviour, or considering the fact that there are emerging third-party firms collecting hundreds of thousands of data points in order to predict audience turnouts according to the creative DNA of a trailer alone. Regardless, it is worth considering how Goldman's words became the go-to industry saying in the first place and why this saying has persisted for as long as it has. I believe that clinging to the Goldman mantra points to a deeper tension inherent to the film and television industry, namely, the tension between art and commerce. Simon and Schroder (2020) accurately describe this tension as developing from "the industry's self-image as promoting creativity as against the imperative to develop and promote products whose appeal can be precisely calculated" (p. 550). There is a kind of idealism embedded in the notion that "nobody knows anything", one that argues that no matter how much 
one attempts to calculate and predict the success of a film or show, creativity, art and the affective experience of the viewer are unquantifiable, and that to be able to pick out the next 'big hit' involves the same kind of creativity required to create it. It's as much about preserving Hollywood's “dream factory" ethos, as it may be about maintaining hierarchical structures and legitimizing the position of highly paid executives. This is why companies like Vault AI, Scriptbook, and Cinelytic repeatedly stress that they value the 'organic' nature of the creative process, positioning their services as platforms for creative assistance only. Because, as Beherens et al. point out, "technologies that challenge the primacy of traditional creative agencies are often feared and critiqued", which is why they tend to be "framed as a decision support tool" (p. 9). Of course, these tactics are only in keeping with the doublespeak typical to the industry, where executives regularly commend the power of creativity and intuition, but strive for financial precision through formulaic creative outputs (e.g. this explains the current dominance of reproduced Intellectual Property in the form of remakes, sequels, and 'universe'based content). For example, Jefferey Katzenberg, former Disney executive and co-founder of DreamWorks Pictures, paraphrased the Goldman adage in an interview in 2016, saying the "crazy thing about the movie business is that there is absolutely no recipe for success" (Mediabiz, 2016). Four years later, he is now the founder of WndrCo., a consumer technology holding company that has launched Quibi, a mobile streaming service that borrows the datacentric business model made popular by the digital native OTT's. Quibi even promotes itself as joining Hollywood with the "best of Silicon Valley", which we can assume includes predictive analytics and AI (Business Wire, 2020).

Ultimately, in this section of my MRP, I have attempted to outline some of the ways Big Data and algorithmic technologies are currently creeping into the most creative practices and elements making up the film and television production process. In doing so, I hope to raise 
questions regarding the shifting nature of creativity in the digital age, as well as draw attention to the difficulty of locating artistic agency in our increasingly networked world. These are currently pressing areas of concern, especially amongst academics who worry that culture itself may be reduced to data and that algorithms could strip creativity of its inherent humanness. However, I would like to conclude this section by offering a less deterministic perspective. First, we must remember that creativity, in the context of the film and television industry, is impossible to divorce from the economic incentives governing it, just as the development of algorithmic technologies are inseparable from the financial motivations tied to their deployment. Creativity within the industry has always been constrained or controlled to varying degrees, with or without algorithms. With that in mind, I believe the current use of Big Data and algorithmic technology for the development of film and television content is not a threat to human creativity as it currently exists within the industry. Instead, it is a by-product of what we can call networked cultural production, in which the very nature of what we find culturally compelling, as well as that of creativity more broadly, is evolving and being spread out across complex networks of meaning-making, and it is doing so in tandem with the tools, tactics and technologies being deployed within the industry. Just as we should conceive of algorithms as being relational, contingent, ontogenetic and "embedded in wider socio-technical assemblages" (Kitchin, 2014, p. 16), we should also conceive as relational the creative process, artistic agency, and the nature of what audiences find affective and compelling today. For example, elements typically understood to make for compelling television might include strong directing, writing, and acting, but this list will increasingly include cross-media characteristics such as the "memeability" of the content or its ability to generate discussion via Twitter, as even the consumption of a single cultural product becomes increasingly dispersed across platforms. Creativity itself has now integrated - and increasingly come to represent - the ongoing and reciprocal process in which cultural content 
and its consumers are divided into countless data points and reconstructed via endless algorithmically generated connections. Arielli (2018) argues that even the creative process of those belonging to the "artworld" (i.e. those making art for art's sake) has now been "blurred in a constant circle where documentation, inspiration and production influence each other" (p. 91). While this recursive and data-centric formulation of creativity certainly warrants retiring the Goldman mantra, its overall significance becomes far more benign if we are to extend the relational perspective I have afforded to algorithms to that of creativity and creative agency itself (Deleuze \& Guattari, 1987). From this perspective, both the material and discursive operations of data analytics and algorithmic technology can be viewed as a development in which the relational and networked nature of creativity has simply been made more tangible and comprehensible via the language of data science and computation. In other words, the tools being used by companies like Vault AI, Scriptbook, and Cinelytic have presented a new materiality for the already recursive and relational nature of creativity. For this reason, I suggest that data analytics and algorithmic technology are best conceived as additional non-human actors profoundly entangled in the creative process of film and television production, as objects "are no mere props for performance but part and parcel of hybrid assemblages endowed with diffused personhood and relational agency" (Vannini, 2015, p. 5). This is not to suggest that these technologies do not have the power to change the nature of creativity and culture, but that their power to do so resides within the network of actors making up these systems and through their associations with them, and therefore should be studied as such.

In this section of my MRP I have explored some of the ways Big Data and algorithmic technology are currently being utilized at the development stage of film and television production, and argued that these technologies are becoming increasingly entangled in the creative process. If this section gave primacy to the perspective of the creator or producer, the 
following section will do so for the consumer or viewer by examining the role Big Data and algorithms currently play in the distribution and consumption of film and television via recommendation systems. 


\section{Exposing the Operational Logics of the Netflix Recommender System}

Objects, by the very nature of their connections with humans, quickly shift from being mediators to being intermediaries, counting for one or nothing, no matter how internally complicated they might be. This is why specific tricks have to be invented to make them talk, that is, to offer descriptions of themselves, to produce scripts of what they are making others - humans or non-humans-do. (Latour, 2005, p. 95)

A core feature of Netflix's business and brand is the Netflix Recommender System (NRS), a collection of proprietary algorithms used to recommend content to users and personalize nearly every aspect of their experience on the platform. As of 2016, the NRS was responsible for approximately $80 \%$ of total hours streamed on Netflix, and the company had valued the combined impact of personalization and recommendation at an estimated 1 billion dollars per year in revenue (Gomez \& Hunt, 2016). In 2016, Netflix expanded globally to an additional 130 countries. This expansion was followed shortly by the launch of an updated version of the NRS capable of operating at a global scale, allowing the system to share data and make recommendations across a total of 190 countries (McAlone, 2016). Unlike the other Big Five streamers, Netflix's financial success depends solely on the company's ability to attract and retain subscribers. Their platform does not funnel users into larger media ecosystems, it is not tied to additional services or products, nor does it generate revenue from advertising. Thus, a central financial incentive for Netflix is the need to prevent existing users from unsubscribing from their service to join competing ones. The NRS plays a crucial role in achieving this, and is currently the most advanced recommendation system offered by any OTT streamer.

As the Streaming Wars progress, recommendation systems like the NRS will become key competitive features for every major OTT streamer, meaning the distribution of film and television, and therefore our consumption patterns, will increasingly be in the hands of semiautonomous algorithmic technologies. This makes the current moment - that just before these systems come to represent the dominant mode of film and television consumption - an important 
one for questioning how they work, what purposes they truly serve, and what their impact on film and television culture has been and will continue to be. As Morris (2015) reminds us, recommendation systems are "far from neutral purveyors of predictions" they "measure and manufacture audiences to provide targeted suggestions for popular cultural goods and exert a logistical power that shapes the ways audiences discover, use and experience cultural content" (p. 447). In other words, they are cultural intermediaries: entities that operate between production and consumption, culture and the economy, constructing symbolic meaning and value around cultural goods and practices, and shaping public tastes and preferences (Maguire $\&$ Matthews, 2014).

Of the many algorithmic applications and practices propagating in the film and television industry, the NRS has received the most scholarly attention. I wish to contribute to this dialogue by continuing to analyze the elements of the NRS that have most typically been called into question, namely, its impact on the distribution and consumption of film and television, as well as its potential implications regarding notions of taste. In order to do so, I have conducted a small experiment, applying Bucher's (2018) method of reverse engineering to the NRS. This allowed me to engage directly with its algorithmic interface in an effort to expose its operational logics. However, before discussing the details of my experiment it is first necessary that I provide an overview of the technical specificities of the NRS, which I have primarily gathered from two sources: a paper published by Netflix computer scientist's Carlos Gomez-Uribe and Neil Hunt titled “The Netflix Recommender System: Algorithms, Business Value, and Innovation," and the Netflix Technology Blog. 


\section{The Netflix Recommender System}

In broad terms, the NRS is powered almost entirely by machine learning, using a combination of content based-filtering and collaborative filtering algorithms to recommend content. Content-based filtering relies solely on a user's past data, which is gathered according to their interactions with the platform (e.g. viewing history, watch time, scrolling behaviour, etc.). In order to produce recommendations and personalize a user's experience this data is combined with other large intricate data-sets that contain information derived from the 15,000 film and television titles offered by Netflix worldwide, including items such as their genre, category, actors, director, and release year (Wasko \& Meehan, 2020). Collaborative filtering involves the same data extraction process, but makes its recommendations according to a weighted combination of other users' preferences, thus imitating person-to-person recommendations. Previously, the NRS's collaborative filtering recommendations were limited to the data extracted from users in a specific region or country (Stenovec, 2016). Now recommendations are pulled from the viewing preferences of users across the world, and users themselves are algorithmically grouped into global "taste communities," of which there are currently over 2000 (Wasko \& Meehan, 2020).

While the NRS is often referred to by the public or the media as the "Netflix algorithm", Gomez and Hunt (2016) point out that this is a vast oversimplification: "Our recommender system is not one algorithm, but rather a collection of different algorithms serving different use cases that come together to create the complete Netflix experience" (p. 2). Some of the more specific algorithms and models used by Netflix include: matrix factorization (used for collaborative filtering), neural networks (used for catalogue organization and pattern recognition), clustering, topic models, Bayesian nonparametrics, and Gaussian processes (Basilico, 2016). These algorithms are used either individually or in combination to create 
multiple proprietary systems of algorithms, which together make up the complete NRS. According to Gomez and Hunt (2016) these main systems making up the NRS include the following: the Personalized Video Ranker (a general purpose system of algorithms that orders and ranks the entire Netflix catalogue into personalized genres and rows); the Top-N Video Ranker (a system used to generate "top picks" according to the head of the catalogue ranking); the Trending Now Ranker (a system for capturing and organizing temporal trends); the Continue Watching Ranker (a system that sorts the subset of recently viewed content according to what a user is most likely to resume watching or re-watch); Video-Video Similarity (an impersonalized algorithm that produces a ranked list of similar titles for every video in Netflix's catalogue, typically displayed in a "Because You Watched" row); Page Generation (a fully personalized system that selects and orders rows according to user relevance and diversity; and Search (a system of algorithms that combine play data, search data, and metadata to optimize search results). According to Netflix, the NRS was built on and operates according to the following principals:

We want our recommendations to be accurate in that they are relevant to the tastes of our members, but they also need to be diverse so that we can address the spectrum of a member's interests versus only focusing on one. We want to be able to highlight the depth in the catalog we have in those interests and also the breadth we have across other areas to help our members explore and even find new interests. We want our recommendations to be fresh and responsive to the actions a member takes, such as watching a show, adding to their list, or rating; but we also want some stability so that people are familiar with their homepage and can easily find videos they've been recommended in the recent past. (Netflix Technology Blog, 2015) 
Crucial to the development of the NRS has been Netflix's consistent use of A/B testing. $\mathrm{A} / \mathrm{B}$ testing is used to measure the effectiveness of various algorithm and recommendation variants through the comparison of control and experimental groups of Netflix users (Gomez \& Hunt, 2016). These groups receive alternate recommendations, displays, and experiences. The control group undergoes the same Netflix experience offered to all members, while the experimental groups receive alternate algorithmic treatments (Netflix Technology Blog, 2016). According to Gomez and Hunt (2016) A/B test results are Netflix's "most important source of information for making product decisions" (p. 11).

Alongside methodical A/B testing, another area Netflix derives its competitive advantage is the sheer granularity of its data, which the NRS transforms, categorizes, evaluates, and uses to produce recommendations. For example, every film or show offered on Netflix has been individually tagged by human experts with a "rich taxonomy of 200 different story data points" (Gomez \& Hunt, 2016). These story attributes include items such as the content's level of romance, goriness, plot conclusiveness, and even the moral status of its characters. Once tagged, content is algorithmically organized into appropriate "thematic containers" i.e. genres and hyperspecific altgenres (Gomez \& Hunt, 2016). Unrealized by most users, Netflix offers approximately 77,000 altgenres (Madrigal, 2014) that group content into as precise categories as "Dark Suspenseful Gangster Dramas", "Cerebral French Art House Movies" and "Visuallystriking Goofy Action \& Adventure."

Since the introduction of the NRS in 2007, the system has evolved from solving a title ratings and ranking problem ${ }^{3}$, to introducing algorithmic page generation, to now operating as the primary tool for maximizing user engagement by personalizing nearly every aspect of the

\footnotetext{
${ }^{3}$ Hallinan and Striphas (2016) provide a detailed account of the "Netflix Prize", which was situated around solving this problem.
} 
Netflix interface and user experience (Chong, 2020). Today, the entire Netflix homepage is algorithmically generated, including all suggested titles, the ranking of those titles within custom "rows" (e.g. "Crime Dramas", "Top Picks", etc.), and the ordering of those rows on the homepage. Titles that the NRS ranks as being most relevant to a user land at the start of a row and those rows determined to be most relevant appear higher on the homepage itself. Furthermore, almost all the information displayed regarding a specific title is personalized including its match score, artwork, trailer, synopsis, and metadata (e.g. awards, cast, etc.). In the words of Netflix their "deep personalization" has enabled the company to "not have just one Netflix product but hundreds of millions of products: one for each member profile" (Netflix, n.a).

\section{Reverse Engineering the Netflix Recommender System}

I have reverse engineered the NRS by setting up three separate Netflix profiles, each designated with its own specific taste persona. For two weeks (14 days), I selected one new film or television show per day, per profile according to each profiles' predetermined taste preferences. I allowed each selected film or show to play in its entirety as the NRS takes into account watch times, fast-forwarding, and exit rates. I also gave each selected title a 'thumbs up', providing the NRS with an additional indication of that persona's preferences. I tracked the results by taking screenshots of the homepage every day, before a new selection was made.

In order to construct these personas, I adapted a method commonly used by advertisers and marketers called consumer personas (see, e.g, Cooper, 1999; Mulder \& Yaar, 2006; Nielsen, 2013). Consumer personas are developed with the intent of producing a realistic character sketch of a specific target audience, which can then be used to guide future marketing and advertising decisions. Mulder and Yaar (2006) explain that "each persona is an archetype serving as a 
surrogate for an entire group of real people" (p. 19). They can be presented as hyper specific personalities (e.g. The Explorer) or as a single fictional person (e.g. Francis the First-Time Home Buyer) (Mulder \& Yaar, 2006, p.20). A consumer persona not only includes a mix of demographic and geographic data, but also psychographic characteristics, i.e. a persona's behaviours, goals, values, preferences, and attitudes (Well, 1975).

I drew on this method to better structure the taste personas used in my own experiment, primarily borrowing from it the use of psychographics and rich character description. However, unlike the consumer personas being used by marketing firms, my Netflix taste personas serve no purpose for directing business decisions. They were developed with the aim of exposing the operational logics of the NRS, and therefore intend to represent different 'types' of Netflix users. Because they were specifically constructed in order to capture the vicissitudes of the NRS, each persona is best thought of as an exaggerated caricature of potentially real Netflix users and were designed with the hope of generating highly dissimilar recommendations. Much like Netflix's use of 'taste communities' (which are proprietary and inaccessible to the public) these personas are archetypes, representative of different Netflix user populations who not only possess varying tastes and preferences, but express different attitudes in regards to why they use Netflix and what compels them to consume film and television.

Three unique Netflix taste personas were constructed, each possessing distinct identities with specific preferences for film and television content. These include the Die-Hard Sports Fan, the Culture Snob, and the Hopeless Romantic. In addition to these three personas, I created a control profile which I have named the Disruptor. This profile was devoid of a persona and was operated according to no taste preferences. I only used this profile in an attempt to disrupt the NRS by making random and usually contradictory content choices. 
The outline for each taste persona contains the following elements: a brief character description; their favourite film; their favourite television show; and three Netflix altgenres that most accurately define their tastes. In order to avoid biases in my own decision-making, I chose to leave out basic demographic information such as age, gender, race, ethnicity, and geolocation. In doing so, the following taste persona's may actually align more closely with Netflix's own use of "taste communities", which the company claims have replaced their previous reliance on more traditional demographic categorizations (Lynch, 2018).

\section{Netflix Taste Personas}

\section{The Die-Hard Sports Fan}

The Die-Hard Sports Fan turns to film and television content to supplement their unbounded passion for live sports. When they're not watching live games, they spend their leisure hours reading athlete biographies, managing fantasy sports teams, and swearing at community centre referees. If it weren't for a mysterious knee injury, the Die-Hard Sports Fan surely would have gone pro, but they are now content to live vicariously through the athletes they see on TV. They turn to sports stories as a source of inspiration valuing the hard work and dedication embodied by their favourite athletes. The Die-Hard Sports Fan enjoys any and all sports related content from golf to basketball, to CrossFit and Big Wave surfing. Outside of sports they enjoy the odd superhero movie, and they make concerted efforts to avoid romantic comedies and anything they deem to be too pretentious. The Die-Hard Sports Fan doesn't bother themselves with notions of taste, and simply views Netflix as just another avenue for satisfying their ceaseless hunger for sports content.

Favourite Film: Rocky

Favourite Show: Friday Night Lights

Netflix Micro Genres: Inspiring Sports Documentaries; Oscar-winning Sports Dramas; and Extreme Sports

\section{The Culture Snob}

The culture snob is a highly educated individual who wants to engage with intellectually challenging and stimulating cultural content, though they claim to dabble in more "low brow" fare from time to time. They wish to spend their downtime strolling through parks, attending art galleries, reading avant-garde literature, and talking at their friends about subjects they have no interest in. The culture snob considers themselves to be a cinephile and takes matters of taste seriously. They seek out obscure, classic, foreign, and critically acclaimed content, and generally tend to be less interested in watching television shows; they particularly despise reality TV. The Culture Snob frequently questions why they even have a subscription to Netflix to begin with, 
which more often than not leads them to long bouts of inner dialogue regarding cogs and machines.

Favourite Film: 81/2 (dir. Federico Fellini)

Favourite Show: HBO's The Wire

Netflix Micro Genres: Critically-acclaimed Independent Movies; Classic Foreign Dramas; and Cerebral Art House Movies

The Hopeless Romantic

The Hopeless Romantic engages with cultural content in order to escape the monotony of everyday life; for them this escape is best facilitated by films and shows teeming with passion, romance, sex, and high drama. Their love for romance is so pure and unfettered that where others may draw lines between high and low culture (e.g. Culture Snob), they make no such distinctions for romantic content; in a single day they may go from being totally engrossed in the details of the latest celebrity wedding, to tearing through the pages of a Jane Austen classic. Outside of romance they tend to prefer musicals or more current and popular content, particularly reality TV. They have zero interest in sports or documentary content. Overall, The Hopeless Romantic doesn't take matters of taste too seriously because for them Netflix is a place to escape into more romantically compelling and dramatic worlds.

Favourite Film: The Notebook

Favourite Show: The Bachelor

Netflix Micro Genres: Steamy Forbidden-Love Dramas; Sentimental Romantic Tearjerkers; and Feel-good Romantic Comedies

\section{Findings}

Upon setting up each profile, I was prompted with an option to jumpstart the NRS by selecting three titles of interest from a list of a few dozen options. I skipped this step for each persona, and as a result they all began with a virtually identical homepage, which according to Netflix represents "a diverse and popular set of titles" (Netflix, n.a). On Day 1 of the experiment, the Die-Hard Sports Fan watched two episodes of Netflix's Michael Jordan docuseries “The Last Dance", the Culture Snob watched Francis Ford Coppola's "The Godfather"; and the Hopeless Romantic watched “Crazy Stupid Love” starring Ryan Gosling and Emma Stone. By day two, each homepage had been slightly reordered by the NRS, alternative artworks had appeared for 
select titles, and one or two altgenre rows populated near the bottom of each profile's homepage. For example, for the Die-Hard Sports Fan, "Formula 1: Drive to Survive" made its way to the first ranked title in the "Netflix Originals" row, "Sports Documentaries" appeared as a genre row, and the altgenre row "Strong Black Male Lead" emerged near the bottom of the homepage, which interestingly would climb closer to the top over the course of the experiment. In comparison, for the Hopeless Romantic the show "You" (about a romantic stalker) was the first ranked title offered in the "Netflix Originals" row, the already existing genres row "Romantic Movies" and "Comedies" climbed closer to the top of the page, and an altgenre row appeared titled "Girls Night In". For the Culture Snob, "Narcos: Mexico" was the top ranked Netflix original, a "Crime Dramas" row emerged at the top of the homepage, and an altgenre row titled "Drug Lords, Mob Bosses, and Gangsters" had materialized. Aside from these changes, the remainder of the homepage consisted of similar content suggestions across each profile, the majority of which were Netflix original content.

As the experiment progressed each profile's homepage became increasingly personalized and recommendations became more accurate. For example, on Day 5 an altgenre row literally titled "Movies for Hopeless Romantics" appeared halfway down the Hopeless Romantic's home page, and a row titled "Critically-acclaimed Auteur Cinema" made its way to the top of the Culture Snob's. By Day 7 even seemingly neutral genre rows such as "Exciting Movies" and "Familiar Favourites" had been highly tailored to each profile. For the Die-Hard Sports Fan the "Exciting Movies" row now consisted of numerous sports related titles such as Crossfit documentary "Redeemed and Dominant" and hockey documentary "Ice Guardians". Meanwhile "Familiar Favourites" for The Hopeless Romantic included almost entirely romantic comedies such as "Friends With Benefits" and "He's Just Not That Into You". Interestingly, this kind of personalization was even visible within the "Popular on Netflix" row, which could easily be 
mistaken as an objective and impersonal collection dedicated to the most popular/viewed content offered on the platform. As it turns out even this row is organized according to user relevancy. For the Culture Snob the top ranked content within the "Popular on Netflix" row would include award-winning Japanese drama "Shoplifters", while for the Die-Hard Sports Fan it was "Antione Griezman: The Making of a Legend", and for the Hopeless Romantic, "Fifty Shades: Freed".

I continued to select appropriate titles for each persona for the remainder of the experiment: "Coach Carter" for the Die-Hard Sports Fan, David Lynch's experimental short "What did Jack Do" for the Culture Snob, "50 First Dates" for the Hopeless Romantic, and so on. After two weeks, each profile was noticeably personalized, and the NRS had appeared to successfully tailor its recommendations to the tastes of each persona. The Die-Hard Sports Fan's homepage was thoroughly covered in sports content, predominantly of the documentary and non-fiction variety. Interestingly, there was an abundance of suggested content tagged as "inspiring". Even two altgenres emerged titled "Inspiring Dramas" and "Inspiring Documentaries". Similarly, the Culture Snob's homepage consisted of dozens of critically acclaimed, foreign, and independent titles. Altgenre rows named "Hidden Gems", "Dramas Based on Books", and "Cerebral Documentaries" occupied prime homepage real-estate, and even some avant-garde titles were being ranked first for standard genre rows. For example, the dizzying art-house film "Lost Highway" by David Lynch was ranked first in the "Thrillers" row. Furthermore, the Culture Snob received a disproportionately greater number of older title suggestions (e.g. "Lawrence of Arabia" released in 1962), and it was the only profile to receive top ranked suggestions containing foreign content. Likewise, the Hopeless Romantic's homepage was ruled by romance and reality television content. Of the three profiles the Hopeless Romantic's homepage had the least diverse offering of titles and rows beyond those 
belonging to their taste preferences (I assume this is because Netflix has a greater number of romance-related content in their catalogue). Included in Figure's 1-3 are examples of rows from each profile's homepage following the 14-day experiment:

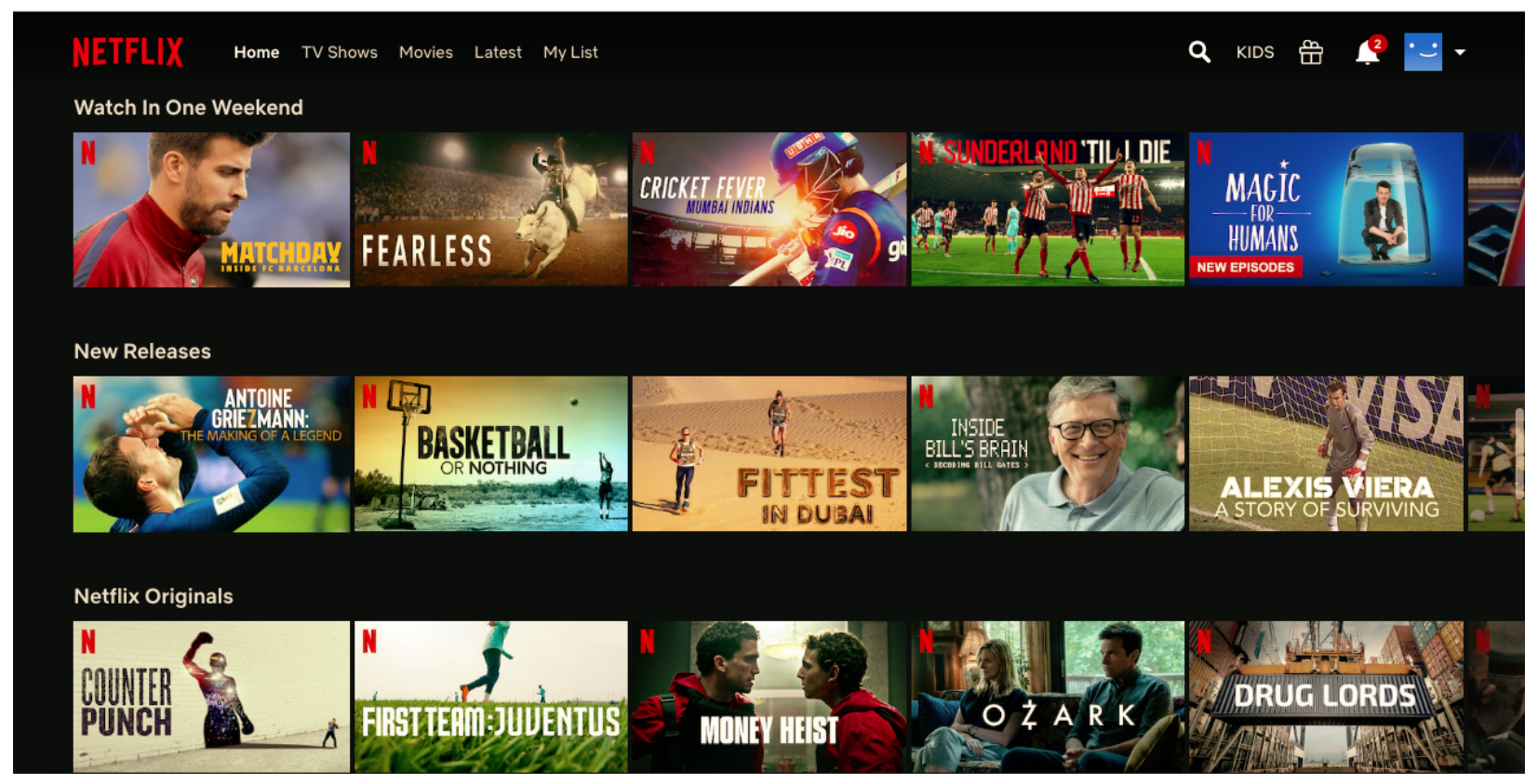

Figure 1: Screenshot of the Die-Hard Sports Fan's homepage following the 14-day experiment.

NETFLIX Home TV Shows Movies Latest My List

Hidden Gems
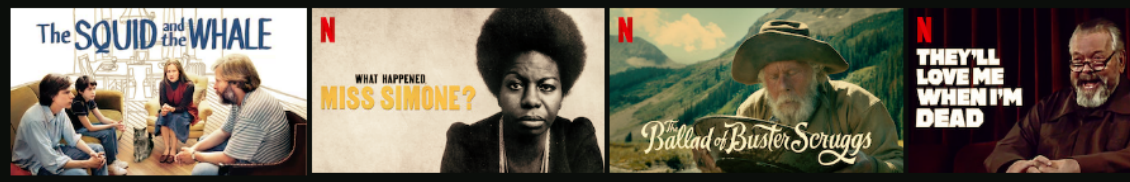

Q KIDS 苗 4

Documentary Films
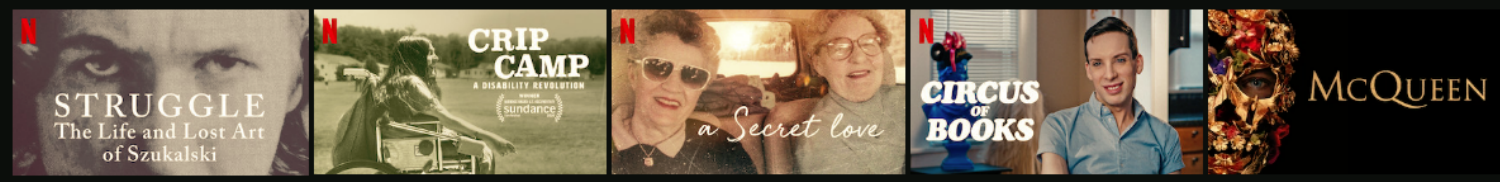

Oscar-winning Dramas >

the social

- network
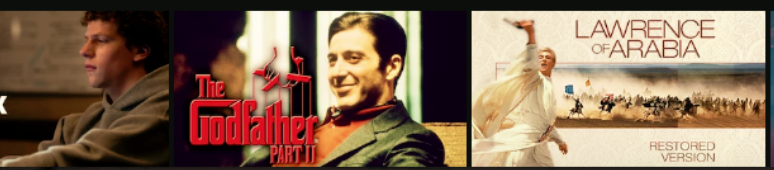

Q IA LA LAND

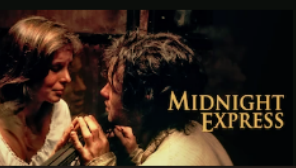

Figure 2: Screenshot of the Culture Snob's homepage following the 14-day experiment. 


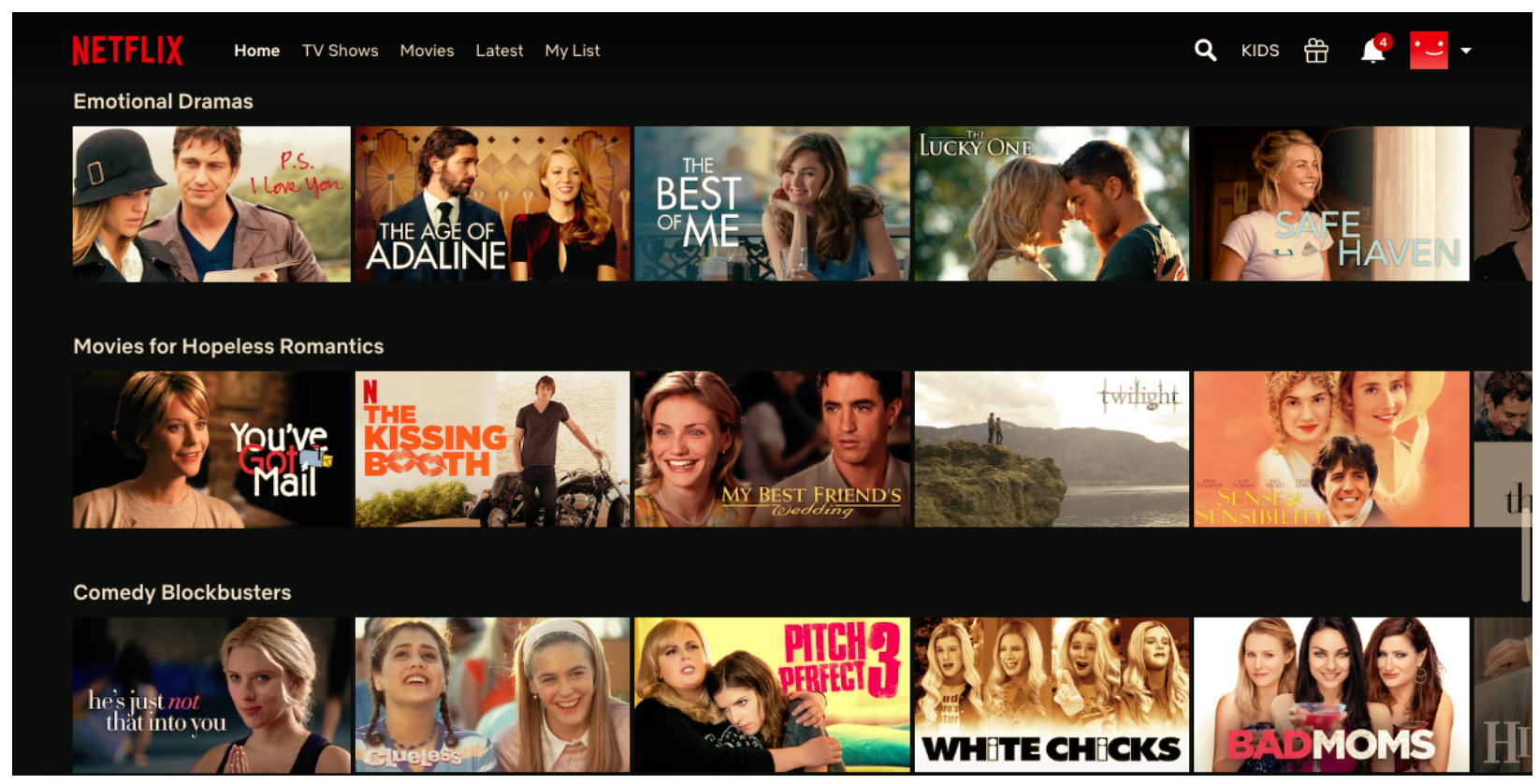

Figure 3: Screenshot of the Hopeless Romantic's homepage following the 14-day experiment.

While the NRS certainly appeared to lock-in on the individual tastes of each persona, it is important to note that not every item appearing on their respective homepage was a direct reflection of their tastes. Of the forty rows offered on each profile, about half were standard and overlapping genre rows such as "Documentaries", "Comedies", and "TV Shows”. However, there was little crossover between the actual content offered within these rows between profiles. For example, the titles suggested within the "Romantic Movies" row were consistently very different between the Hopeless Romantic and the Culture Snob's homepages. That being said, there were significant similarities in content recommendations amongst outlier rows i.e. those that appear unrelated to the taste preferences of a specific persona (e.g. "Action Movies" offered to the Hopeless Romantic). These rows presented what seemed to be a generic collection of the same "diverse and popular titles" suggested by the NRS on Day 1 of the experiment. This is likely evidence of the NRS's effort to highlight the "depth" and "breadth" of Netflix's catalogue 
for the purpose of "helping members explore and discover new interests" (Netflix Technology Blog, 2015). Alternatively, it could be seen as proof that Netflix favours certain titles and pushes their own original content onto users despite knowing a user's individual preferences.

Some of the most interesting and unexpected results of the experiment came from changes in title artwork. The NRS's artwork personalization did end up making each persona's homepage quite visually distinct, even when taken as whole. For example, by the end of the experiment The Hopeless Romantic's homepage was occupied by a disproportionate number of images of men and women romantically embracing. Of the first 10 titles suggested for the Hopeless Romantic, which were spread across the top two rows, five of them presented artwork images containing a romantic embrace (e.g. a couple kissing or staring into one another's eyes). Additionally, the banner image at the top of the homepage was a blown-up still of Brad Pitt and Claire Forlani kissing, taken from the film "Meet Joe Black". Likewise, the Die-Hard Sports Fan's homepage was covered with artwork images containing movement (e.g. running, jumping, catching, racing, etc.), and they also generally displayed brighter and more electric colours. In addition, there was a significant number of shirtless bodies and athletic physiques dispersed across the rows. Meanwhile the Culture Snob's homepage - though the subtlest of the three was dominated by darker hues, black and white artwork images, and plenty of actor headshots. Taken as a whole, these changes did create a sensation of entering and scrolling through distinct aesthetic worlds via the homepage. However, the most noteworthy changes in artwork were those differences between artwork images used for the same title across all three profiles. By the end of the experiment, when identical titles were offered to each profile, they almost always had different artwork images, though I did not document any differences in trailers or synopses. Not only were artwork images for the same titles different from one another across profiles, but they were personalized quite accurately for each taste persona. For example, at one point, the Netflix 
original series "Outerbanks" was recommended for all three profiles. The show follows a group of teenagers living on the Outer Banks of North Carolina, who band together in an attempt to discover a legendary treasure that is tied to the disappearance of the protagonist's father. The artwork image presented for "Outberbanks" on the Culture Snob's homepage was of the protagonist, Chase, facing the camera atop a collaged backdrop containing a map and a still image from the show. However, for the Hopeless Romantic it was a close-up of Chase and a female character, Madelyn, about to kiss. For the Die-Hard Sports Fans the artwork image was two male characters carrying surfboards as they walk into water. While the artwork image for the Culture Snob appeared quite neutral, the other two had been accurately personalized to the Hopeless Romantic and the Die-Hard Sports Fan's specific tastes. I have included a comparison of these artwork images below (see Figure 4), as well as those presented for the film " $\mathrm{La} \mathrm{La}$ Land", which were also tailored differently to each profile, for what appear to be similar reasons.
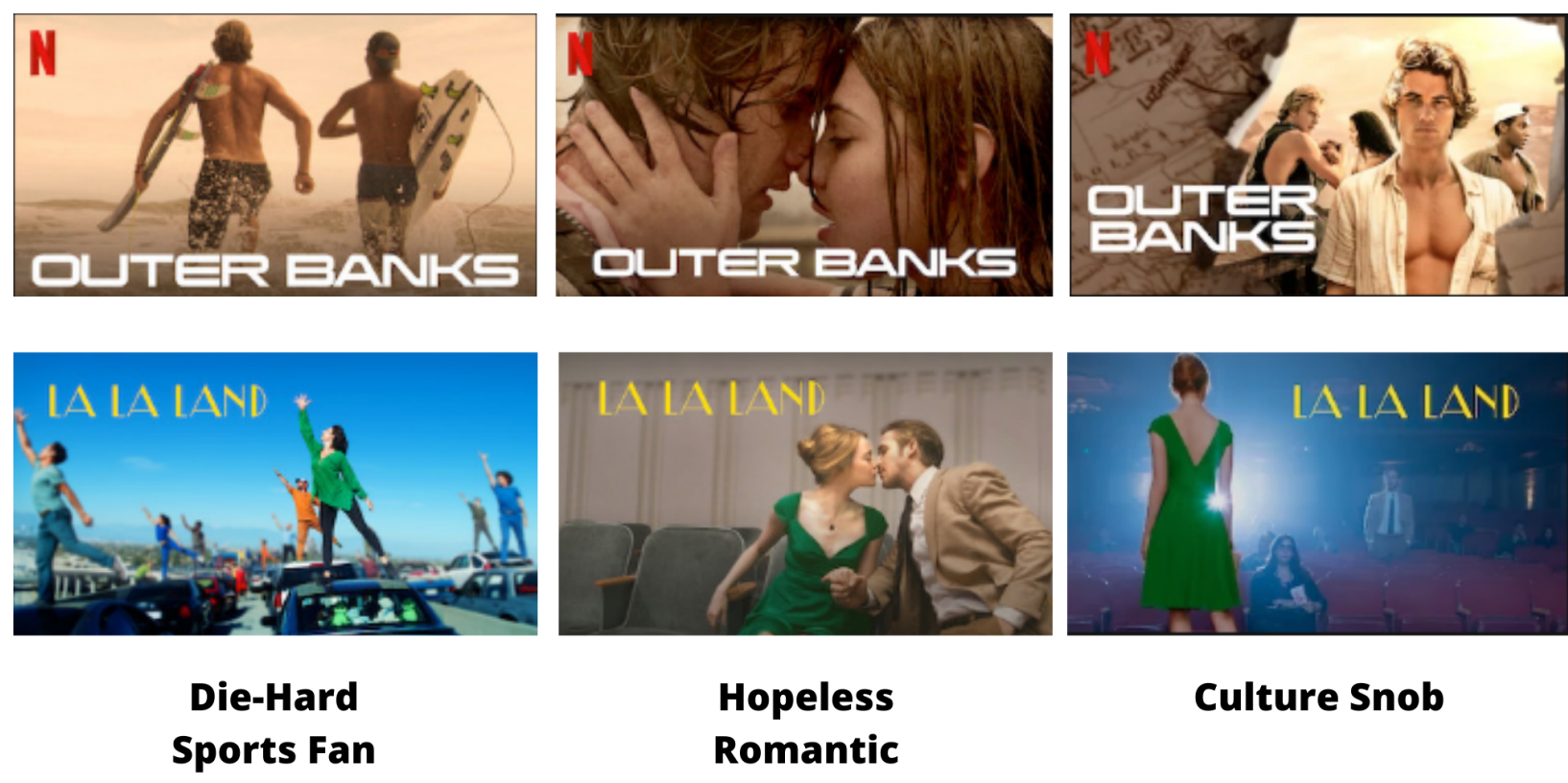

Romantic

Figure 4: Comparison of artwork personalization for "Outberbanks" and "La La Land" for each taste persona. 
Lastly, I want to briefly discuss the Disruptor's homepage. For the Disruptor I did not follow the same two-week schedule as the other personas. Rather, over the course of the month of May, 2020 I irregularly deployed bursts of content viewing, selecting films and shows that I believed would be most conflicting with one another. For example, I had the Disruptor watch multiple episodes of the romance reality show, "Love Is Blind", followed immediately by Orson Welles' 1941 classic “Citizen Kane”. In addition, I thumbed up and down dozens of titles, whether they had been watched or not. Again, I tried to keep these intentionally inconsistent. For example, "Kill Bill :Volume 1" would receive a thumbs up, but "Kill Bill: Volume 2" would get a thumbs down; "Shrek" would get a thumbs up, but so would the "Irishman", followed by a thumbs down for "Incredibles 2" and "The Godfather". For the most part, this seemed to lead to a highly diverse homepage absent of an easily distinguishable taste identity. However, the chaotic selecting of content did appear to produce a small rift in the NRS. An "Exciting Films" row was consistently suggested at the top of Disruptor's homepage. The row contained six of the eight "Fast and the Furious" films offered on Netflix, of which were all ranked as the top ten options within the row (see Figure 5). The remaining two films in the series were also located elsewhere on the homepage.

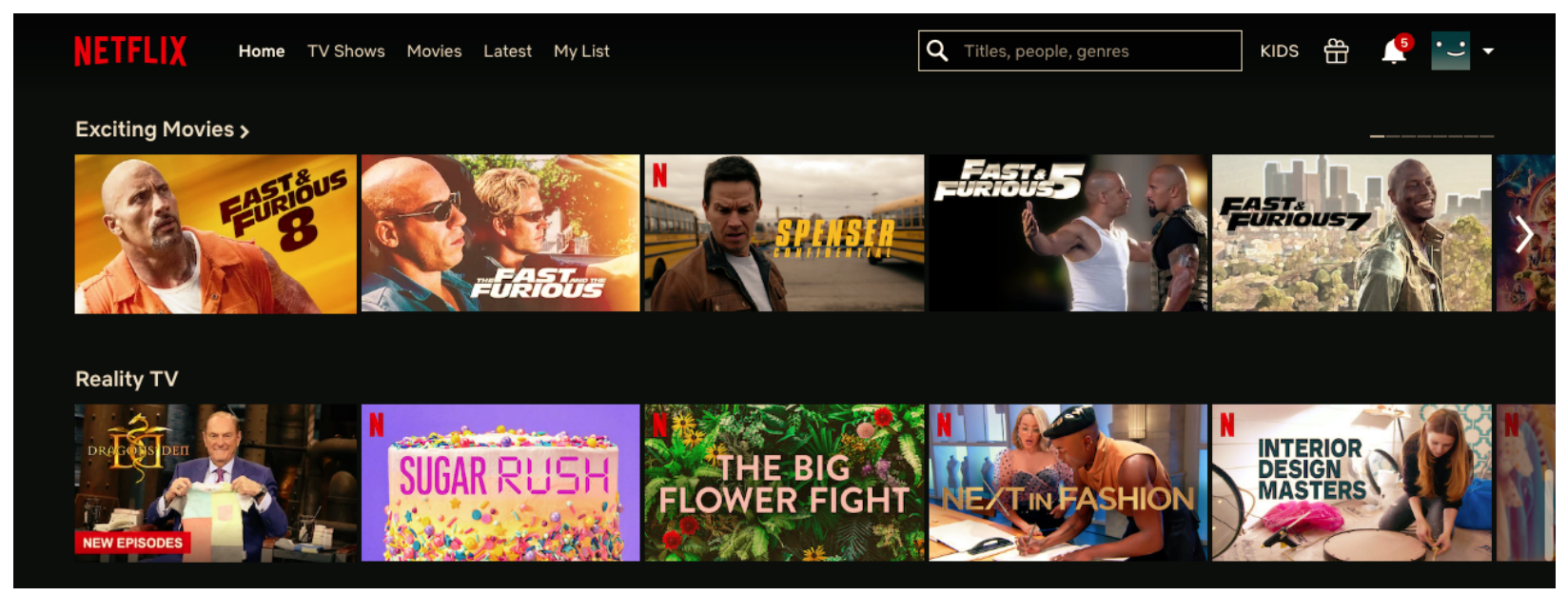

Figure 5: Four of the first five titles offered in the Disruptor's "Exciting Movies" row belonged to the "Fast \& Furious" film series. 


\section{Analysis}

\section{The Circular and Economic Logic of the Netflix Recommender System}

By the end of the experiment each profile had been individually reconfigured and thoroughly personalized via the algorithmic operations of the NRS. Rows were reordered, precise altgenres had materialized, artwork images changed, and a seemingly endless - as well as ever-changing slate of content titles had populated, all tailored to the exact tastes and preferences of each persona. No doubt, this model of user-centric personalization has made Netflix an exemplary case of the way culture - in today's algorithm-saturated world - "finds us" (Beer, 2009), rather than the other way around.

Not surprisingly, what my experiment had made particularly obvious was the anticipatory and circular logic embedded in the NRS and all of its operations. In order for a Netflix user to experience the level of "deep personalization" outlined above, only two things are required of them: consumption and interaction. The more content a user consumes on Netflix and the more time they spend interacting with the platform itself (e.g. scrolling, clicking, rewinding, searching, etc.), the more data the NRS is able to extract from them, resulting in more elaborate personalizations and increasingly precise recommendations for both them and the global Netflix community via collaborative filtering. In theory, a more personalized experience, as well as more accurate recommendations should then lead to greater user consumption and interaction, and therefore more data, better recommendations, and so on and so forth. Nowhere in this circular equation is it necessary for users to seek out new content, search for specific titles, or experiment across genres. For example, by Day 3 of my experiment and onwards, each persona would have hardly had to venture past the second row of their homepage in order to consume their preferred content ad infinitum. Of course, this is a concern frequently raised in regards to all algorithmic recommender systems, namely, that of feedback loops and filter 
bubbles, which in the case of the NRS would allow for what Arielli (2018) has called "algorithmic self-confirming aesthetic consumption" (p. 86). The concern with this kind of consumption is that feedback loops only reinforce a user's pre-existing preferences, diminishing their exposure to a diverse range of cultural offerings, and denying art, aesthetics, and culture of its confrontational societal role. Furthermore, there is concern that NRS-generated feedback loops threaten to homogenize film and television culture, particularly considering that the individual feedback loops of each user can "iteratively influence the collaborative filtering algorithm's predictions over time" (Sinha et al, 2016). What this means is that the individual feedback loops each user is caught in (e.g. the Die-Hard Sports Fan's endless loop of sportsrelated content) can contribute to large-scale, even global loops, because user consumption patterns are extracted by the NRS and contribute to recommendations made to other users across the world.

However, when thinking about the feedback loop phenomena and its cultural implications, we must be hesitant to work from the assumption that the NRS reproduces a user's true tastes in the first place. Netflix does not sell content in the traditional sense, it sells itself as a platform that can be purchased for a monthly subscription fee. Unlike services such as YouTube, Netflix makes no money from advertising revenues or single transactional sales (TVOD). Therefore, content is leveraged by Netflix only as a means of attracting and retaining users (retention being an increasing priority for all OTT streaming services amidst the Streaming Wars). Gomez and Hunt (2016) explain that in order to improve Netflix's retention rates, you must increase user engagement, which is where the NRS truly derives its business value:

If we create a more compelling service by offering better personalized recommendations, we induce members who were on the fence to stay longer, and improve retention. In addition, all members with an improved experience (not just those on the fence) may be 
more enthusiastic when describing Netflix to their friends, strongly influencing new subscriber acquisition through word-of-mouth effects. (p. 8)

Therefore, it is crucial to recognize that Netflix's deployment of the NRS in order to "present the right content" to every user according to their specific tastes (what it is advertised to do) is not the same as deploying the NRS to maximize user engagement (what it actually does). Content that keeps users coming back is not always reflective of their tastes, nor is a homepage that promotes interaction always personal. As Arieli (2018) points out "it would be naïve to think that the goal of a successful platform would be to simply mirror our actual preferences", as in reality recommendation systems like the NRS "are private tools aimed at maximizing profit through increased user engagement.” (p. 89). For example, when discussing Netflix's use of A/B testing to guide their product decisions, Gomez and Hunt (2016) explained that "the main measurement target of changes to our recommendation algorithms is improved member retention" (p. 9). Nowhere do the authors mention the measurement of user satisfaction, nor is this something discussed in Netflix's blog post on the same subject. ${ }^{4}$ What this means is that the accuracy of the NRS to predict user tastes is simply likened to increases in user engagement, effectively equating consumption with authentic taste performance. Thus, from a research perspective, we must not take what the NRS selects to be relevant and meaningful for each user at face value, just as we must not view the NRS as a black-boxed technical device operating independently from culture. Deeply embedded in the NRS is a purely economic reasoning that was carefully designed by software engineers, who were guided by Netflix executives. This moves the problem of feedback loops beyond the concerns associated just with "self-confirming

\footnotetext{
${ }^{4}$ See “It's All A/Bout Testing: The Netflix Experimentation Platform”, Netflix Technology Blog. https://netflixtechblog.com/its-all-a-bout-testing-the-netflix-experimentation-platform4e1ca458c15\#: : :text=Overview,Netflix) $\% 20$ which $\% 20$ receive $\% 20$ alternative $\% 20$ treatments.\&text $=$ This $\% 20$ cell $\%$ 20represents $\% 20$ the $\% 20$ control,members $\% 20$ not $\% 20$ in $\% 20$ the $\% 20$ test.
} 
aesthetic consumption" to one regarding the implications of being trapped in "closed commercial loops" (Hallinan \& Striphas, 2016, p. 122).

Interestingly, my experiment revealed that the "closed commercial loops" produced by the NRS extend beyond the reproduction of consumption patterns and into the entire aesthetic experience of navigating the Netflix interface. The personalization of artwork, trailers, synopses, and metadata can be attributed to what Netflix calls 'evidence selection' algorithms. These algorithms alter the way content is presented in order to provide each user the appropriate 'evidence' that a recommended show or film is right for them (Gomez \& Hunt, 2016). Netflix claims by personalizing artwork specifically they, "help each title put its best foot forward for every member and thus improve our member experience" (Netflix Technology Blog, 2017). However, as my experiment progressed and each persona's homepage became increasingly visually personalized, it was never clear how artwork customization could translate to improved user experiences. For example, it seemed to be far more misleading than accommodating when the artwork image for "Good Will Hunting" changed for the Hopeless Romantic from an image of Robin Williams to one of Matt Damon and Minnie Driver romantically embracing. For the Hopeless Romantic, "Good Will Hunting” would simply appear to be one of the dozens of romantic movies displayed on their homepage, especially considering it was surrounded by other romantic titles with comparable artwork. Similarly misleading was the selecting of an image of surfboarders for a television show that was not about surfing (i.e. "The Outerbanks" as displayed to The Die Hard-Sports Fan), or displaying black and white images for films shot in full-colour (as seen on the Culture Snob's homepage). What again became clear was the economic motivations of the NRS, in this case, its use of "evidence selection" specifically. Personalized artwork coupled with lengthy homepages and rows built for endless scrolling creates the illusion that Netflix contains an infinite amount of content perfect for every user. This illusion is easily 
conceivable when considering the affective quality of images, which, when absorbed dozens at a time, could register at an unconscious level, subtly shaping a user's perceptions of the Netflix experience. For example, if the Die-Hard Sports fan was to casually scroll down their homepage, by the time they reached the bottom they would have been inundated with a plethora of sports related imagery, whether they were completely aware of it or not. They also would have passed dozens of half visible, yet possibly relevant titles fading out on the right-hand side of every row. Whether unconsciously perceived or consciously noted, personalization via "evidence selection" would likely incite feelings in a user that no matter how much of their favourite content they have consumed, they have only scratched the surface of Netflix's offerings. Though these changes in artwork may at first seem trivial they could ultimately be the reason many users renew their subscriptions every month, which is why Netflix has introduced the feature in the first place. Thus, "evidence selection", I would argue, is almost entirely a tool designed to improve retention rates, not user experience, making it a prime example of what Sandvig (2014) has called "corrupt personalization": a personalization scheme built around a user's preferences and presented as if to be in their best interest, but which really serves the goals of the provider of the scheme and does so at the expense of the user.

Another example of this kind of "corrupt personalization" witnessed during my experiment - and perhaps one that is more directly illustrative of the NRS's built-in economic reasoning - was the consistent recommendation of the "Fast \& Furious" films for each of my experiment's personas. On June $3^{\text {rd }}$, Netflix Canada added the first seven films from the "Fast \& Furious" franchise to their platform, in addition to already offering its eighth installment (Hynes, 2020). This meant that Netflix Canada now offered eight of the nine "Fast \& Furious" films available. Considering these were all hugely popular films when released, Netflix's licensing of the series is a strong example of the kind of 'tent-pole' content strategy currently defining the 
Streaming Wars. Throughout my experiment the "Fast \& Furious" films were recommended to each persona, despite never matching their tastes. By the end of the experiment two films from the franchise were being recommended in outlier rows on both the Die-Hard Sports Fan and the Hopeless Romantic's homepages. While this could be attributed to the NRS's calculated promotion of discovery-based content (Netflix Technology Blog, 2015), the fact that the Disruptor's homepage was so overwhelmingly oriented towards promoting this content seemed uncoincidental. By the end of the experiment all eight of the "Fast \& Furious" films available on Netflix appeared on the Disruptor's homepage, six of which were presented in a single top ranked row titled "Exciting Movies". As mentioned earlier, these recommendations did not appear as a result of the Disruptor watching action films or other related content. Rather, they seemed to have emerged in this unusual way due to a rift or glitch in the NRS caused by an excessive, random, and contradictory selection and 'thumbing' of content. As Striphas (2015) and Bucher (2016) have pointed out, it is within these moments of malfunction that the logics of algorithms tend to reveal themselves most plainly. In this case, it appeared that when the NRS was overwhelmed with difficult and inconsistent input data it defaulted to recommending content with a high likelihood of producing user engagement, and did so under the guise of personalization. Not only is each "Fast \& Furious" film likely to produce a high degree of user engagement due to their known popularity, but taken together they represent a 'bingeable' series making them particularly useful for increasing retention rates. Furthermore, they could be leveraged to funnel users into watching Netflix's own original "Fast \& Furious" spin-off series titled "Fast and Furious Spy Racers". Perhaps, what this rift in the system exposed was a specific commercial feedback loop constructed around the "Fast \& Furious" series itself. Combined, these eight films would take users over 16 hours to watch, and considering user retention is the 
current currency of the Streaming Wars, it would be in Netflix's best interest to have users watch every second of them - or better yet, plan to eventually do so.

\section{Recommendation Systems, Agency, and Taste-Making}

My analysis of the NRS and its operational logics is nested in a broader inquiry regarding the tension between human and algorithmic agency as it relates to cultural consumption and recommendation systems. It is not entirely clear if the NRS is predicting what film and television content users may prefer, or whether it is determining it; and it is within this murky territory of locating agency where many critical questions about recommendation systems have been raised. Has the NRS passed the "threshold" as Finn (2017) puts it from "modeling to building cultural structures"? And if so, what does it mean that the NRS operates according to a circular and economic logic? It is nearly impossible to engage with these questions without taking into consideration notions of taste, as it is our tastes that are understood to drive our cultural consumption patterns and vice versa.

As a cultural intermediary the NRS plays a critical role in the process of film and television taste-making. It reveals and conceals various titles and genres, and exercises control over our "decision pathways" (Finn, 2017, p. 97). More specifically, as Gaw (2019) has pointed out, the NRS constructs taste within a "social vacuum" relying solely on data generated within and by the recommender system, and it undertakes the "quantification of taste" by "splicing" users and content into "infinite attributes to create taste communities and altgenres" (pp. 39-40). This algorithmic construction of taste by the NRS is precisely what several scholars have criticized (see, e.g., Striphas 2015, Gaw 2019). For them, the question of agency goes beyond whether the NRS is dictating what we watch, to whether it is a prime example of the ways algorithms are now exerting control over our artistic and aesthetic judgements (i.e. our cultural 
preferences) - which we tend to believe are inherently human and deeply personal affairs - and doing so, according to logic of computation. For example, Hallinan and Striphas (2016) question the implications of a "court of algorithmic appeal" in which "objects, ideas, and practices are heard, cross-examined, and judged independently, in part, of human beings" (p. 129).

However, if we are to adopt a relational materialist perspective of algorithms we must diverge from the typical critical readings of the NRS and recommendation systems like it. According to a relational ontology, agency itself is inherently distributed; it is a mediated phenomenon brought to fruition via the associations of a multiplicity of both human and nonhuman actors (Latour, 2005), or as Barad (2003) sees it, "agency is not an attribute but the ongoing reconfigurings of the world" and the universe itself is "agential intra-activity in its becoming" (p. 818). Thus, the relational perspective is not compatible with the critical readings typically leveraged against recommendation systems, because it does not attribute individual agency to algorithms, nor to humans in regards to the development of their 'personal' tastes and preferences. In accepting this position, the dialogue surrounding the relationship between the NRS and taste-making takes a drastically different turn. Therefore, I want to suggest the adoption of Hennion's (2004) "pragmatic" conception of taste as it coincides with the relational nature of algorithms that I have promoted throughout this paper. In doing so, I am consciously stepping away from Bourdieu's (1984) influential theory of taste, which has been drawn on previously to support the critical social analysis of recommendation systems (see, e.g., Morris, 2015; Gaw, 2019 ).

According to Hennion, taste is a reflexive and performative activity with a deeply "entangled history" that is always in the process of unfolding; it is "corporated, framed, collective, equipped" and simultaneously produces both the taster and the objects they value (Hennion, 2004, pp. 133, 131). Hennion presents his theory as a direct criticism of Bourdieu and 
his follower's perspective on taste, which he views as restrictive and reductive. For Bourdieu (1984) taste is socially constructed. It is acquired and refined according to a person's social and cultural capital. Notions of 'good taste' and 'bad taste' are not inherent, but learned through social conditioning and are constructed in accordance to the dominant group or ruling class's choosing. Because taste is directly tied to social class, it acts as an instrument for reproducing hierarchies between and within class groups, and therefore, tastes are only falsely perceived to be unique and personal (Maguire \& Matthews, 2014).

Though Hennion (2004) agrees with Bourdieu's proposition regarding the relational nature of taste, he rejects the theory for presenting a "radically unproductive" view that mistakenly reduces cultural objects to mere "random signs" and positions tasters as "passive subjects of an attachment" who are ignorant to the true determinants of their passions (i.e. their social position) (p. 131). Alternatively, he argues that taste has the capacity to "transform sensibilities and create new ones", because people are inherently active and productive: they "constantly transform objects and works" (Hennion, 2004, p. 131). Furthermore, he argues that Bourdieu's conception of taste refuses to take the "amateur" seriously: the passionate fan or cultural virtuoso who does not belong to the social elite, but who may collect with other amateurs to form their own rules and hierarchies of knowledge surrounding works of pop culture or those deemed to be 'low-brow'; these amateurs, Hennion argues, are guided by an identical relational process of taste-making as those of the social elite. Hennion's analysis of taste places emphasis on the reciprocal relationship between cultural works and their audience, seeing taste as a "co-formation of a set of objects and the frame of their appreciation" (p. 134). This model of taste requires "even more ties, attachments, and mediations" as every incremental step involved in tasting (i.e. the conscious consuming of cultural products) influences both "future perceptions and past catalogues of works" (p. 134). 
If we accept Hennion's "pragmatic" conception of taste, we accept that we do not have absolute agency over our tastes and preferences, that they are not completely derived from the aesthetic properties of the works we consume, nor can they be explained away as being the result of "external determinisms" tied to our social position (Hennion, 2001, p. 1). From a relational perspective, taste, like algorithms, is performative and transformative, it exists and functions in a constant state of revision, where its cultivation and purpose is negotiated amongst the various actors involved in its becoming. These actors can include, the individual taster, their social position, their personal temperament and disposition, the commercial market, the cultural work and its various aesthetic elements, and now the algorithmic technologies ordering and ranking these works according to their own circular and economic logics; logics which are designed by engineers, who are guided by business executives, who themselves are informed by market researchers relying on other systems of algorithms. Therefore, the NRS is best conceived of as one of the many actors deeply entwined in the complex networks that constitute contemporary film and television taste-making, adding profound complexity to notions of taste by introducing an additional layer of abstraction via the language, logics, and interfaces of computation. Moreover, the NRS and its algorithmic operations appear to actually mirror the relational, reflexive, and performative way taste exists and operates in the world more broadly, giving it material form by compounding key elements typically involved in the construction of taste into a single and highly accessible digital platform. Considering the NRS constructs taste in a social and cultural vacuum (Gaw, 2019), it could further be viewed not just as a reflection, but a microcosm of the relational, reflexive, and performative processes of taste-making as described by Hennion. For example, Hennion (2004) argues that taste is "a performance: it acts, engages, transforms and is felt" (p. 131). On Netflix, taste is not ascribed to inherently passive users according to the social or demographic dimensions of their profiles. Rather, users perform taste 
through consumption and interaction, which the NRS continuously tracks, measures, and leverages in order to transform nearly every aspect of Netflix, including, those user's profiles and experiences, the global ranking of Netflix's existing catalogue, and the development of future content. Transformation is, I would argue, the fundamental labour of the NRS, and taste on the platform can only be performative; it is always being "formed as it is expressed and is expressed as it is formed" (Hennion, 2005, p. 135). This means that Netflix and its users reciprocally construct one another, as agency over taste passes between consumer, producer, and every actor in-between. Furthermore, Hennion (2004) argues that taste is "accomplished through a collective which provides a frame" (p. 135). In the case of the NRS, the collective includes all of Netflix's global users now spanning 190 countries, who cultivate each other's tastes via collaborative filtering algorithms, which are framed in Netflix's algorithmically generated homepage. Hennion points out that even the methods regulating the construction of taste (e.g. the mainstream media, critics, blogs, etc.) are constantly being revised and updated. The Netflix microcosm puts this notion into overdrive, as the NRS's algorithmic methods for governing taste are continuously being revised and optimized via nonstop A/B testing.

Ultimately, if we are to adopt a relational materialist approach to studying the NRS we must reject the commonly posed dichotomy between human and algorithmic agency, which often dominates the discussion around recommendation systems and their influence on tastemaking. In doing so, the NRS should no longer be positioned as an unknowable technical device capable of dictating our experience of culture, as its agency over our tastes and preferences, much like our own, remains only partial. In fact, as I have described above, the NRS could perhaps more usefully be viewed and studied as microcosm for the relational, reflexive, and performative way taste operates in the world more broadly, giving these complex processes a new computational materiality. 


\section{Conclusion}

Going farther from the shore, the deep waters of algorithmic imagination draw us relentlessly back toward ourselves and the mysterious origins of cognition, inspiration, and serendipity that drive creative work. (Finn, 2017, p. 185).

This MRP has set out to accomplish two interrelated goals: 1) to explore the current role algorithms play in the production, distribution, and consumption of film and television, and to assess how these technologies are impacting broader notions of creativity and taste within the industry; and 2) to push back against the critical theoretical perspectives that have come to dominate the discourse surrounding algorithmic cultures. In order to do so, I have joined Bucher $(2016$; 2018) in adopting a relational materialist perspective of algorithms. In addition, I have extended the relational ontology I have afforded to algorithms to the concepts of creativity, taste, and culture, as these are equally complex and fundamentally relational entities in and of themselves. I have argued that algorithms must not be conceived of as static, opaque, technical "black boxes" that exert power and control over cultural life, while remaining detached from culture itself. Rather, algorithms are dynamic and evolving socio-technical processes that come into existence and operate in the world via a series of complex relations between human and non-human actors. They are contingent, ontogenetic, performative, and reflexive in nature and are "embedded in wider socio-technical assemblages" (Kitchin, 2017, p. 16). They simultaneously shape and are shaped by the complex network of actors constituting their existence, making their purpose, power, agency, and activity, inherently distributed. Following this description, it becomes clear that algorithms are not just entangled with culture, but that they are culture.

To be clear, in adopting a relational perspective of algorithms, I am in no way rejecting the notion that algorithms are now exerting influence over our cultural practices, ideas, and realities. I have simply argued that the power for them to do so is not top-down nor 
unidirectional. Instead, the power of algorithms to influence creativity, taste, and culture resides within the network of actors making up these systems and through their associations with them. Therefore, to only consider the ways creativity, taste, and culture are being replaced or weakened by algorithmic technology is to ignore the myriad of other actors involved in their operations, most importantly, that of humans; the designers and developers of these technologies, the users providing them the necessary input data they need to function, and the collective that together articulate, discuss, and contest their existence, as the algorithm's presence in everyday life becomes increasingly 'felt', but not necessarily understood. In adopting a relational perspective of algorithms, the question becomes how might the very meanings of creativity, taste, and culture be expanding to accommodate for these new technologies and what ways could they help us understand ourselves and our culture. This framing effectively broadens the discussion, compelling us to ask critical questions that move beyond 'what ifs' regarding whether algorithms are replacing some fundamental aspect of human creativity, or whether they are now dictating our 'personal' tastes and preferences. Instead, we can begin to question why we appear to be increasingly orienting ourselves and our cultural practices towards algorithms and how our conceptions and practices of creativity, taste, and culture may be evolving alongside them.

I have suggested throughout this paper that we must think critically and carefully about the ways the relational nature of algorithms (as described above) may actually reflect, more than disrupt, the cultural practices and concepts that we may be concerned that these technologies are subsuming, such as creativity, taste, and culture itself. We must assume that these technologies are at least, in part, mapping and modeling our cultural reality. Consider for a moment that film and television critics are now using the term 'algorithmic' as a pejorative in their reviews, where they may have used 'formulaic' or 'generic' before. David Ehrlich (2020), senior critic at 
Indiewire, justified giving Netflix's romantic comedy series “The Lovebirds" a one star review due to its being "flat and algorithmic". Does this shift in language prove that algorithms have effectively infiltrated the realm of creativity and storytelling? Or is it an example of language being updated to describe a pattern that has always existed, but has now been made more visible via the language, logics, and processes of computation? Taking the latter's line of argumentation a step further, let us momentarily choose to engage with one of the philosophical tenets of computationalism, the idea that reality itself may be fundamentally algorithmic to begin with. This is the argument that computer scientist and physicist Stephen Wolfram laid out his groundbreaking book, A New Kind of Science. Wolfram (2002) argues that all complex systems - those inherent to our biology, our physical world, our social and cultural processes, even questions of consciousness - can effectively be mapped via computation, because the universe itself is fundamentally computational in nature. Wolfram proposes a new science, like physics or biology, in which scientists systematically, empirically, and experimentally explore the "the computational universe," which is the source of abstract rules that he calls "simple programs" whose problem-solving capabilities surpass those of mathematics. Wolfram's new science looks to fill the need for a more bottom-up scientific method: instead of building theories based on observations post hoc, Wolfram suggests we enumerate "simple programs," let them run, and match their computational patterns with those we observe in the natural world (Wolfram, 2002). For Wolfram, the sea of abstraction that is the "the computational universe" will help us answer fundamental questions about reality. In a blog post written 15 years after the release of his influential book, Wolfram claims that:

A central lesson of A New Kind of Science is that there's a lot of incredible richness out there in the computational universe. And one reason that's important is that it means that 
there's a lot of incredible stuff out there for us to 'mine' and harness for our purposes. (Wolfram, 2017)

Throughout this paper I have posited the need for a less deterministic, more multilayered and relational approach to thinking about algorithms and their current role within the film and television industry. That being said, my research did reveal some potentially negative impacts these technologies could be having on creativity and taste-making. So, allow me to now offer a necessary dose of cynicism.

As algorithmic technology becomes increasingly ubiquitous, an ever-thickening layer of computational abstraction will continue to be draped over all of social and cultural life, which will certainly introduce new ways of framing reality, the least of which will likely be direct reflections of what is already there. As Finn (2017) points out "like Turing's original abstraction machine, these [algorithms] extend a symbolic logic into the cultural universe that reorders minds and meanings that come into contact with them" (p. 54). If my research has revealed one thing, it's that a profound commercial and economic orientation is deeply embedded in the “symbolic logics" carried by systems such as Vault AI's DeepAudience Analysis or the Netflix Recommender System. I would even argue that the fundamental mode of abstraction used by these systems is one that turns creativity, taste, and culture into a problem that can, and must, be efficiently solved. In the case of taste-making, the circular and economic logics embedded in the NRS appear to turn taste into an equation, where authentic taste performance is equated with consumption, and maximizing user engagement and retention rates is considered the end goal. As streaming becomes the dominant mode of film and television consumption, and recommendation systems similar to the NRS begin to proliferate, I'd like to suggest that our notions and practices of taste - both on and offline - will increasingly abide by these logics. The same is true for creativity. Companies like Vault AI, ScriptBook, and Cinelytic, have automated 
the highly creative - and ideally, speculative and experimental - practices of ideation, screenwriting, and casting, abstracting away the reality of these processes through terabytes of data, algorithmically generated connections, sleek user interfaces, and clickable icons. Cinelytic has literally transformed the work of talent agencies, which involve auditions, meetings, brainstorming sessions, and a great deal of intuition, into an abstracted simulation akin to fantasy sports - the difference being decisions made on Cinelytic influence and shape real-world outputs. What becomes clear is that algorithms are not problematic in themselves. In fact, for someone like Wolfram, they are the key to unlocking our future potential as a species. The problem then is the way in which the layer of computational abstraction they have introduced, which now blankets most of cultural reality, has been co-opted or hijacked by corporate and commercial interests. This is particularly clear in the marketing rhetoric and symbolism around recommendation systems like the NRS, where companies like Netflix and Spotify promote their recommendation systems as providing users an avenue for authentic cultural discovery, endless choice, and personal freedom. Paradoxically, it could equally be argued that these systems threaten to homogenize our interactions with culture and force us to outsource our freedom of choice and personal autonomy to algorithms, which we wilfully assume know us better then we know ourselves. Cohn (2018) drives home this point, arguing:

These industries operate through a profound display of doublespeak wherein conformity becomes autonomy, oppression becomes liberty, consumerism becomes empowerment, autonomy becomes collaboration, stagnation becomes happiness, and recommendations become transcendent potentialities. ( p. 187)

Ultimately, algorithms and Big Data will continue to present new abstract frameworks for representing cultural reality, many of which will be overcoded by the commercial and economic interests of those developing and deploying these technologies. However, what I have 
attempted to make clear throughout this paper is that this does not mean we should make it our sole effort as social science and humanities researchers to criticize and tear down these frameworks, as they also present to us new and useful ways of 'seeing' the complex processes inherent to cultural life. For example, in 1986, Hennion and Meadel called the radio, "an invisible machine for making the world visible to itself" (p. 287). Perhaps this is true for all of computation, algorithms and Big Data alike. If so, it would appear that the real concern is that the "computational universe" that Wolfram describes - which could be explored for answers to some of humankind's most profound questions - has, much like any other vital resource, been infiltrated by, and put in service of, private and commercial interests in order to maximize profits rather than human creative capacities. 


\section{References}

Alexander, J. (2019, November 1). Apple TV Plus is basically free because Apple's end game is replicating Amazon Prime. The Verge. https://www.theverge.com/2019/11/1/20942474/appletv-plus-amazon-prime-video-price-bundle-music-arcade-news-services

Alexander, J. (2020, April 21). AT\&T is making HBO Max free to millions of wireless, video, and internet customers. The Verge. https://www.theverge.com/2020/4/21/21229474/hbo-max-attfree-wireless-internet-cable-video-trial-price-launch-date

Ang, I. (2006). Desperately seeking the audience. Routledge.

Arielli, E. (2018). Taste and the algorithm. Studi Di Estetica, O(12), Article 12. http://mimesisedizioni.it/journals/index.php/studi-di-estetica/article/view/628

Arnold, M. ((1993 [1869])). Culture and anarchy and other writings. Cambridge; New York: Cambridge University Press.

Ball, M. (2020, February 10) The flaws of "Subscription Fatigue”, "SVOD Fatigue”, and the "Streaming Wars." https://www.matthewball.vc/all/misnomers

Ball, M. (2019, November 11) The mining of media (or the "Streaming Wars" are just a battle). MatthewBall.Vc. https://www.matthewball.vc/all/minedmedia

Barnes, B. (2012, October 21). Disney, struggling to find its digital footing, overhauls Disney.com. The New York Times. https://www.nytimes.com/2012/10/22/business/media/disney-strugglingto-find-its-digital-footing-overhauls-disneycom.html

Barnes, B. (2019, November 18). The streaming era has finally arrived. Everything is about to change. The New York Times. https://www.nytimes.com/2019/11/18/business/media/streaminghollywood-revolution.html

Barnes, B. (2020, April 8). Disney plus racks up 50 million subscribers in 5 months. The New York Times. https://www.nytimes.com/2020/04/08/business/disney-plus-50-million-subscribers.html

Barad, K. (2003). Posthumanist performativity: Toward an understanding of how matter comes to matter. Signs, 28(3), 801-831. http://www.jstor.org/stable/10.1086/345321

Barad, K. (2007). Meeting the universe halfway: Quantum physics and the entanglement of matter and meaning. Durham, NC: Duke University Press.

Basilico J. (2016, September 18). Recommending for the world [Presentation]. https://www.slideshare.net/justinbasilico/recommending-for-the-world-66144921

Beer, D. (2009). Power through the algorithm? Participatory web cultures and the technological unconscious. New Media \& Society, 11(6), 985-1002.

https://doi.org/10.1177/1461444809336551 
Beer, D. (2013). Algorithms: shaping tastes and manipulating the circulations of popular culture. In D. Beer, Popular Culture and New Media (pp. 63-100). London: Palgrave Macmillan.

Bennett, J., Cheah, P., Orlie, M. A., Grosz, E., Coole, D., \& Frost, S. (2010). New materialisms: Ontology, agency, and politics. Durham, NC: Duke University Press.

Braidotti, R. (2006). Posthuman, all too human towards a new process ontology. Theory, Culture \& Society, 23(7-8), 197-208. https://doi.org/10.1177/0263276406069232

Britton. (2017, January 18). Brainstorming, brainwriting, ideation, and the structured process of idea generation. Medium. https://medium.com/memorable-tv-commercials/brainstormingbrainwriting-ideation-and-the-structured-process-of-idea-generation-3a488abdb667

Bucher, T. (2016). Neither black nor box: Ways of knowing algorithms. Innovative methods in media and communication research, 81-98. https://doi.org/10.1007/978-3-319-40700-5_5

Bucher, T. (2018). If... then: Algorithmic power and politics. Oxford University Press.

Buck, A. M., \& Plothe, T. (Eds.). (2019). Netflix at the Nexus: Content, Practice, and Production in the Age of Streaming Television. Peter Lang Inc., International Academic Publishers.

Buder, E. (2017, April 21) Can AI Predict a Movie’s Success? Algorithmic Screenplay Service "Scriptbook" Causes Major Backlash. (2017, April 21). No Film School. https://nofilmschool.com/2017/04/scriptbook-black-list-screenwriting-ai-algorithm

Burroughs, B. (2019). House of Netflix: Streaming media and digital lore. Popular Communication, 17(1), 1-17.https://doi.org/10.1080/15405702.2017.1343948

Bursztynsky, J. (2020b, April 21). HBO Max will be free for some AT\&T subscribers when it launches on May 27. CNBC. https://www.cnbc.com/2020/04/21/hbo-max-to-launch-may$\underline{27 . h t m l}$

Business Wire. (2020, April 2). T-Mobile Customers Get Quibi on Us! Original Movie-Quality Content Designed for Mobile. Business Wire.

https://www.businesswire.com/news/home/20200402005493/en/T-Mobile-Customers-QuibiUs\%21-Original-Movie-Quality-Content

Business Wire. (2019, February 19). MOVIO data says audience for 2019 OSCAR $®$ "Best Picture" nominated films was youngest and most racially diverse in recent years. Business Wire. https://www.businesswire.com/news/home/20190219006107/en/MOVIO-Data-Audience-2019OSCAR $\%$ C2\%AE-\%E2\%80\%9CBest-Picture\%E2\%80\%9D

Business Wire. (2020, January 8). Data driver Cinelytic engages Warner Bros. Pictures International to utilize their revolutionary AI-driven content and talent valuation system. Business Wire. https://www.businesswire.com/news/home/20200108005856/en/Data-Driver-Cinelytic-EngagesWarner-Bros.-Pictures 
Campo-Rembado M. \& Oakley, S. (2018, October 29). How 20th Century Fox uses ML to predict a movie audience. Google Cloud Blog. https://cloud.google.com/blog/products/ai-machinelearning/how-20th-century-fox-uses-ml-to-predict-a-movie-audience/

Chong, D. (2020, May 2). Deep dive into Netflix's recommender system. Medium. https://towardsdatascience.com/deep-dive-into-netflixs-recommender-system-341806ae3b48

Cohn, J. (2019). A Brief History of Good Choices. In J. Cohn, The Burden of Choice: Recommendations, Subversion, and Algorithmic Culture (pp. 27-53). Rutgers University Press.

Collin, R. (2019, February 15). Out with auteurs, in with the algorithm: How data analysis rewrote the script. The Sydney Morning Herald. https://www.smh.com.au/entertainment/movies/outwith-auteurs-in-with-the-algorithm-how-data-analysis-rewrote-the-script-20190130$\underline{\text { hlamvm.html }}$

Cooper, A. (2004). The inmates are running the asylum: Why high-tech products drive us crazy and how to restore the sanity (Vol. 2). Sams Publishing.

Crisp, V. (2015). Film distribution in the digital age: Pirates and professionals. Palgrave Macmillan. https://doi.org/10.1057/9781137406613

Curtin, M., Holt, J., \& Sanson, K. (2014). Distribution revolution: Conversations about the digital future of film and television. University of California Press.

Deleuze, G., \& Guattari, F. (1987). A thousand plateaus. Minneapolis: University of Minnesota Press.

Deloitte (2020, June 23) Digital media trends 14th edition - press release. Deloitte. https://www2.deloitte.com/us/en/pages/about-deloitte/articles/press-releases/digital-mediatrends.html

Diakopoulos, N. (2014). Algorithmic accountability reporting: On the investigation of black boxes. Digital Journalism, 3(3), 398-416. https://doi.org/10.7916/D8ZK5TW2

Dodge, M., \& Kitchin, R. (2009). Software, Objects, and Home Space. Environment and Planning A, 41(6), 1344-1365. https://doi.org/10.1068/a4138

Ehrlich, D. (2020, May 20). 'The Lovebirds' Review: Kumail Nanjiani and Issa Rae Are Exes on the Run in Algorithmic Netflix Rom-Com. IndieWire. https://www.indiewire.com/2020/05/thelovebirds-review-kumail-nanjiani-issa-rae-netflix-rom-com-1202232283/

Erbland, K. (2020, April 24). Streaming wars: Virtual cinemas offer haven for cinephiles and struggling theaters alike. IndieWire. https://www.indiewire.com/2020/04/virtual-cinema-guide$\underline{1202226844 /}$

Feiner, L. (2019, November 13). Disney+ surpasses 10 million sign-ups since launch. CNBC. https://www.cnbc.com/2019/11/13/disney-surpasses-10-million-sign-ups-since-launch.html 
Finn, E. (2017). What algorithms want: Imagination in the age of computing. MIT Press.

Friedman, K (2017, April 17). How data convinced Netflix to double down on Adam Sandler. PMG Blog. https://www.pmg.com/blog/data-convinced-netflix-double-adam-sandler/

Galloway, A. R. (2006). Gaming: Essays on algorithmic culture (Vol. 18). University of Minnesota Press.

Gaw, M. F. (2019). Algorithmic logics of taste: Cultural taste and the Netflix recommender system [Master's Thesis]. Faculty of Arts and Social Sciences, The University of Sydney. https://ses.library.usyd.edu.au/bitstream/handle/2123/21530/Dissertation\%20final_Redacted.pdf ?sequence $=3$ \&isAllowed $=y$

Gillespie, T. (2016a). \# trendingistrending: When algorithms become culture. In R. Seyfert, \& J. Roberge, Algorithmic cultures: essays on meaning, performance and new technologies (pp. 64-87). Routledge.https://doi.org/10.4324/9781315658698

Gillespie, T. (2016b). Algorithm. In B. Peters (Ed.), Digital Keywords: A Vocabulary of Information Society and Culture, 18-30. https://ebookcentral-proquest-com.ezproxy.lib.ryerson.ca

Goldman, W. (1983). Adventures in the screen trade. New York: Warner Books.

Gomez-Uribe, C. A., \& Hunt, N. (2016). The Netflix Recommender System: Algorithms, business value, and innovation. ACM Transactions on Management Information Systems, 6(4), 1-19. https://doi.org/10.1145/2843948

Greenberg, J. (2015, December 11). Netflix is using Adam Sandler to beat Hollywood and rule the world. Wired. https://www.wired.com/2015/12/netflix-ridiculous-six-adam-sandler-to-beathollywood-and-rule-the-world/

Hallinan, B., \& Striphas, T. (2016). Recommended for you: The Netflix Prize and the production of algorithmic culture. New Media \& Society, 18(1), 117-137. https://doi.org/10.1177/1461444814538646

Hayes, D. (2020, May 13). What Price Streaming? For Newest Players Like HBO Max, It's Not A Simple Equation. Deadline. https://deadline.com/2020/05/streaming-price-war-hbo-max-disneyplus-apple-subscribers-1202932889/

Hennig-Thurau, T., \& Houston, M. B. (2018). Entertainment science: Data analytics and practical theory for movies, games, books, and music. Springer, Cham. https://doi.org/10.1007/978-3-319$\underline{89292-4}$

Hennion, A. (2004). Pragmatics of taste. The Blackwell Companion to the Sociology of Culture, 131144. https://halshs.archives-ouvertes.fr/halshs-00193146

Hennion, A., \& Meadel, C. (1986). Programming music: Radio as mediator. Media, Culture \& Society, 8(3), 281-303. https://doi.org/10.1177/016344386008003003 
Hsieh, C.-K., Campo, M., Taliyan, A., Nickens, M., Pandya, M., \& Espinoza, J. J. (2018). Convolutional Collaborative Filter Network for Video Based Recommendation Systems. ArXiv:1810.08189 [Cs]. http://arxiv.org/abs/1810.08189

Hynes, H. (2020, May 20). The 'Fast \& Furious' movies 1-7 dropped On Netflix so get ready for a wild wide. Narcity. https://www.narcity.com/entertainment/ca/fast-and-furious-movies-1-7-areavailable-on-netflix-right-now

Introna, L. D., \& Hayes, N. (2011). On sociomaterial imbrications: What plagiarism detection systems reveal and why it matters. Information and Organization, 21(2), 107-122. https://doi.org/10.1016/j.infoandorg.2011.03.001

Jarvey, N. (2019, October 16) Disney over the top: Bob Iger bets the company (and Hollywood's future) on streaming. The Hollywood Reporter.

https://www.hollywoodreporter.com/features/bob-iger-bets-company-hollywood-s-futurestreaming-1247663

Kay, J. (2019, February 1). How data company Cinelytic aims to reduce risk in the film business. Screen Daily. https://www.screendaily.com/features/how-data-company-cinelytic-aims-toreduce-risk-in-the-film-business/5136245.article

Kelly, J. (2019). Television by the numbers: the challenges of audience measurement in the age of Big Data. Convergence, 25(1), 113-132. https://doi.org/10.1177/1354856517700854

Kitchin, R. (2017). Thinking critically about and researching algorithms. Information, Communication \& Society, 20(1), 14-29. https://doi.org/10.1080/1369118X.2016.1154087

Kidwell, E. (2020, February 26) Is CBS All Access free with a cable subscription? CordCutters. https://www.cordcutters.com/cbs-all-access-free-cable-subscription

Lazar, N. (2014). The arts_-digitized, quantified, and analyzed. In M. Pitici (Ed.), The Best Writing on Mathematics 2014, 5, 96-104. https://doi.org/10.1515/9781400865307-010

Littleton, E. \& Low, C. (2019, December 17). Adapt or Die: Why 2020 Will Be All About Entertainment's New Streaming Battleground. Variety.

https://variety.com/2019/biz/features/streaming-2020-disney-plus-netflix-hbo-max-apple-tvamazon-1203439700/

Lynch, J. (2018, July 29). Netflix thrives by programming to 'Taste Communities,' not demographics. Ad Week. https://www.adweek.com/tv-video/netflix-thrives-by-programming-to-tastecommunities-not-demographics/

Madrigal, A. C. (2014, January 2). How Netflix reverse-engineered Hollywood. The Atlantic. https://www.theatlantic.com/technology/archive/2014/01/how-netflix-reverse-engineeredhollywood/282679/

Mager, A. (2012). Algorithmic ideology: How capitalist society shapes search engines. Information, Communication \& Society, 15(5), 769-787. 


\section{https://doi.org/10.1080/1369118X.2012.676056}

Maguire, J. S., \& Matthews, J. (2014). The Cultural Intermediaries Reader. SAGE.

McAlone, N. (2014, June 14). Why Netflix thinks its personalized recommendation engine is worth \$1 billion per year. Business Insider. Retrieved June 23, 2020, from https://www.businessinsider.com/netflix-recommendation-engine-worth-1-billion-per-year$\underline{2016-6}$

McAlone, N. (2016, June 2). Amazon CEO Jeff Bezos said something about Prime Video that should scare Netflix. Business Insider. https://www.businessinsider.com/amazon-ceo-jeff-bezos-saidsomething-about-prime-video-that-should-scare-netflix-2016-6

McKelvey, F., Tiessen, M., \& Simcoe, L. (2015). A consensual hallucination no more? The Internet as simulation machine. European Journal of Cultural Studies, 18(4-5), 577-594. https://doi.org/10.1177/1367549415584856

Mediabiz (2016, March 3). Jeffrey Katzenbergs Gedankenspiele um Paramount. Mediabiz. https://goo.gl/Fn1TNA.

Movio. (n.d.). Movio, shaping the future of movie marketing. Movio. https://movio.co/

Mulder, S. \& Yaar, Z. (2006). The user is always right: A practical guide to creating and using personas for the web. New Riders.

Nielsen, L. (2013). Personas - User Focused Design, Springer Science \& Business Media. Springer.

Netflix (n.a). How Netflix's recommendations system works. Netflix Help Center. https:/help.netflix.com/en/node/100639

Netflix Technology Blog (2017, April 19). It's all A/Bout testing. Medium. https://netflixtechblog.com/its-all-a-bout-testing-the-netflix-experimentation-platform$\underline{4 \mathrm{e} 1 \mathrm{ca} 458 \mathrm{c} 15}$

Netflix Technology Blog (2017, April 19). Learning a personalized homepage. Medium. https://netflixtechblog.com/learning-a-personalized-homepage-aa8ec670359a

Newitz, A. (2016, September 6). Movie written by algorithm turns out to be hilarious and intense. Ars Technica. https://arstechnica.com/gaming/2016/06/an-ai-wrote-this-movie-and-its-strangelymoving/

Raustiala, K., \& Sprigman, C. J. (2019). The second digital disruption: Streaming and the dawn of data-driven creativity. New York University Law Review, 94, 1555-1622. http://dx.doi.org/10.2139/ssrn.3226566

Roberge, J., \& Seyfert, R. (2016). What are algorithmic cultures? In J. Roberge, \& R. Seyfert, Algorithmic cultures: essays on meaning, performance and new technologies (pp. 1-25). Routledge. https://doi.org/10.4324/9781315658698 
Sandvig, C. (2014). Corrupt personalization. Social Media Collective Research Blog, 26. https://socialmediacollective.org/2014/06/26/corrupt-personalization/.

Sandvig, C., Hamilton, K., Karahalios, K., \& Langbort, C. (2014). Auditing algorithms: Research methods for detecting discrimination on internet platforms. Data and discrimination: converting critical concerns into productive inquiry, 22. https://pdfs.semanticscholar.org/b722/7cbd34766655dea10d0437ab10df3a127396.pdf

Seaver, N. (2017). Algorithms as culture: Some tactics for the ethnography of algorithmic systems. Big Data \& Society, 4(2), 1-12. https://doi.org/10.1177/2053951717738104

ScriptBook. (n.d.). ScriptBook. https://www.scriptbook.io

ScriptBook. (2018, November 5). Man and Machine: AI as (co)-creator in storytelling. Medium. https://blog.scriptbook.io/man-and-machine-ai-as-co-creator-in-storytelling-537e5995ea88

Siegel, T. (2020, January 8). Warner Bros. signs deal for AI-Driven film management system (exclusive).The Hollywood Reporter. https://www.hollywoodreporter.com/news/warner-brossigns-deal-ai-driven-film-management-system-1268036

Simon, F. M., \& Schroeder, R. (2020). Big data goes to Hollywood: The emergence of big data as a tool in the American film industry. Second International Handbook of Internet Research, 549567. https://doi.org/10.1007/978-94-024-1555-1_63

Singh S. (2020). Why am I seeing this?. New America. http://newamerica.org/oti/reports/why-am-iseeing-this/

Sinha, A., Gleich, D. F., \& Ramani, K. (2016). Deconvolving Feedback Loops in Recommender Systems. In D. D. Lee, M. Sugiyama, U. V. Luxburg, I. Guyon, \& R. Garnett (Eds.), Advances in Neural Information Processing Systems 29 (pp. 3243-3251). Curran Associates, Inc. http://papers.nips.cc/paper/6283-deconvolving-feedback-loops-in-recommender-systems.pdf

Smith, M. D., \& Telang, R. (2016). Streaming, sharing, stealing: Big data and the future of entertainment. The MIT Press.

Smith, M. D., \& Telang, R. (2018, January 23). Data can enhance creative projects-just look at Netflix. Harvard Business Review. https://hbr.org/2018/01/data-can-enhance-creative-projectsjust-look-at-netflix

Social Media Collective (2016). Critical algorithm studies: A reading list. https://socialmediacollective.org/reading-lists/critical-algorithm-studies/

Spangler, T. (2020, January 16a). Netflix projected to spend more than $\$ 17$ billion on content in 2020. Variety. https://variety.com/2020/digital/news/netflix-2020-content-spending-17-billion12034692371 
Spangler, T. (2020, February 19b). Traditional pay-TV operators lost record 6 million subscribers in 2019 as cord-cutting picks up speed. Variety. https:/variety.com/2020/biz/news/cable-satellitetv-2019-cord-cutting-6-million-1203507695/

Spangler, T. (2020, April 21). Netflix Packs on Record 16 Million Subscribers in Q1, Getting Huge Lift From Coronavirus Lockdowns. Variety. https://variety.com/2020/digital/news/netflixrecord-16-million-subscribers-q1-2020-coronavirus-1234586125/

Stenovec, T. (2016, February 17). Netflix has discovered something profound about people around the world. Business Insider. https://www.businessinsider.com/netflix-global-recommendationsystem-2016-2

Stiff, D. (2019, January 23). The true concept drivers for Aquaman's global box office dominance. Vault AI Blog. https://www.vault-ai.com/posts/aquamans-global-box-office-dominance.html

Storyfit, (n.d.) Storyfit. https://www.storyfit.com/

Striphas, T. (2014, April 30). Algorithmic Culture. Culture now has two audiences. A conversation with Ted Striphas Medium. https://medium.com/futurists-views/algorithmicculture-culture-now-has-two-audiences-people-and-machines-2bdaa404f643

Striphas, T. (2015). Algorithmic culture. European Journal of Cultural Studies, 18(4-5), 395-412. https://doi.org/10.1177/1367549415577392

Striphas, T., \& Hallinan, B. (2016). Recommended for you: The Netflix Prize and the production of algorithmic culture. New Media \& Society, 18(1), 117-137.

https://doi.org/10.1177/1461444814538646

The Atlantic (n.d). Big data and Hollywood: A love story. (n.d.). The Atlantic. http://www.theatlantic.com/sponsored/ibm-transformation-of-business/big-data-and-hollywooda-love-story/277/

The Economist (2020, March 27) Covid-19 is a short-term boon to streaming services.. The Economist. https:/www.economist.com/graphic-detail/2020/03/27/covid-19-is-a-short-termboon-to-streaming-services

Thompson, S. (2018, December 23). “Aquaman" flounders: Where its opening weekend box office ranks in the DCEU. Forbes. https://www.forbes.com/sites/simonthompson/2018/12/23/aquaman-flounders-where-itsopening-weekend-box-office-ranks-in-the-dceu/

Travers, B. (2020, March 1). Streaming wars report: Netflix top 10 lists build buzz, Dick Wolf looks to the future. IndieWire. https://www.indiewire.com/2020/03/streaming-services-ranked-netflixtop-10-dick-wolf-peacock-1202214379/

Tribbey, C. (2020, March 11). Cinelytic CEO looks to make most of P2P piracy data. Media \& Entertainment Services Alliance. https://www.mesalliance.org/2020/03/11/cinelytic-ceo-looksto-make-most-of-p2p-piracy-data/ 
Uricchio, W. C. (2015). Recommended for you: Prediction, creation and the cultural work of algorithms. The Berlin Journal, 28, 6-9.

Van Dijck, J., \& Poell, T. (2016). Understanding the promises and premises of online health platforms. Big Data \& Society, 3(1), 1-11. https://doi.org/10.1177/2053951716654173

Vannini, P. (2015). Non-representational methodologies: Re-envisioning research. Routledge.

Vault AI (n.d). Vault AI. http://www.vault-ai.com/

Vincent, J. (2020, January 9). Warner Bros. signs AI startup that claims to predict film success. The Verge. https://www.theverge.com/2020/1/9/21058094/ai-film-decision-making-warner-brossigns-cinelytic

Wasko, J., \& Meehan, E. R. (2020). A Companion to television. John Wiley \& Sons.

Wells, W. D. (1975). Psychographics: A critical review. Journal of marketing research, 12(2), $196-$ 213. https://doi.org/10.1177/002224377501200210

Whitehead, A. N. (1978). Process and reality: An essay in cosmology Ed. David Ray Griffin and Donald W. Sherburne, NY: Free Press.

Wolfram, S. (2002). A new kind of science. Wolfram Media.

Wolfram, S. (2017). A new kind of science: A 15-Year view. Stephen Wolfram Writings. https://writings.stephenwolfram.com/2017/05/a-new-kind-of-science-a-15-year-view/

Youtube (2017, February 27). You know what's cool? A billion hours. Official YouTube Blog. https://youtube.googleblog.com/2017/02/you-know-whats-cool-billion-hours.html 